\title{
Minimum time optimal control simulation of a GP2 race car
}

\author{
December 2017 \\ Dal Bianco Nicola (nicola.dalbianco@dii.unipd.it) \\ Lot Roberto (roberto.lot@soton.ac.uk) \\ Gadola Marco (marco.gadola@unibs.it)
}

\begin{abstract}
In this work optimal control theory is applied to minimum lap time simulation of a GP2 car, using a multibody car model with enhanced load transfer dynamics. The mathematical multibody model is formulated with use of the symbolic algebra software MBSymba and it comprises 14 degrees of freedom (dof), including full chassis motion, suspension travels and wheel spins. The kinematics of the suspension is exhaustively analysed and the impact of tyre longitudinal and lateral forces in determining vehicle trim is demonstrated. An indirect optimal control method is then used to solve the minimum lap time problem. Simulation outcomes are compared with experimental data acquired during a qualifying lap at Montmeló circuit (Barcelona) in the 2012 GP2 season. Results demonstrate the reliability of the model, suggesting it can be used to optimize car settings (like gearing and aerodynamic setup) before executing track tests.
\end{abstract}

\section{Introduction}

Lap time simulation of racing vehicles are nowadays widely used to predict car performance on race tracks and to optimise the vehicle setup before track tests. The two most common methods used to perform such kind of simulations are represented by the quasi steady state and optimal control approaches. In the former, a fixed trajectory is provided as input data, then the path is divided into small segments and the vehicle maximum speed is calculated at each corner apex. Starting from these known points, acceleration and braking zones are reconstructed by forward and backward integration. Examples of quasi steady state simulations can be found in $(1 ; 2 ; 3 ; 4)$. These kinds of simulations are relatively fast to compute and also very robust, even when highly detailed car models are utilised. For this reason, quasi steady state simulations are the most used by race teams as performance-optimising tools; an example is the Dallara Lap Time Simulation (DLTS) software (5). The fixed trajectory represents a limitation for the accuracy of the optimisation output.

Optimal control calculus instead allows to find simultaneously all driver inputs that maximise the performance (usually the minimum lap time) and either the trajectory can be obtained as a result of the simulation or a fixed driving line can be imposed. However, this type of simulations is significantly more difficult to solve due to the complexity of the optimisation problem, thus the car model is often simplified. The first relevant works in this area came at the beginning of the new millennium by Casanova, $(6 ; 7 ; 8)$, who adopted a non-linear programming (NLP) optimisation algorithm to solve the minimum time problem of a 7 dof car model for long manoeuvres (full laps). The car model used comprised 4 dof for the wheel spin and 3 dof for the chassis, which are the longitudinal, lateral and yaw motion. Since the model does not include any suspension degree of freedom (dof), tyre loads are calculated with in quasi steady state (QSS) conditions using 4 balance equations. This car model is still widely used in recent works, 
as it can be found in $(9 ; 10 ; 11 ; 12 ; 13)$. Kelly in $(9)$ presented a newer NLP algorithm to compute the minimum manoeuvring time both for few turns, and for a full lap, with different car models. The first, and simplest one, is the same model of Casanova (6). The second one adds the suspension travel and tyre loads are dynamically calculated from tyre radial deformation (suspensions are modelled as vertical springs). The third one is similar to the first but adds a thermodynamic tyre model. Another work by the same author can be found in (14), where almost the same car models are used. Recently, other authors have used a simpler single track car model $(15 ; 16)$; however these two works do not aim at solving the minimum time problem, but at demonstrating the advantage of the handbrake technique in particular scenarios. Moreover, a full high fidelity Pacejka Magic Formula Tyre model (17) has been used. In all the above cited optimal-control-related works $(6 ; 7 ; 8 ; 9 ; 10 ; 13 ; 14 ; 15 ; 16)$, except for $(11)$ and (12), drag and lift coefficients are constant. However, in the case of GP2 or F1 car, the aerodynamic forces depend significantly on the ride heights, thus any car model for such car category should include a more complex aerodynamic modelling. In (11), a full lap of a Formula1 car on the Montmeló circuit is simulated, and aerodynamic forces are obtained through a pre-calculated map as function of tyre loads, while in (12) the aerodynamic coefficients depend on the forward speed only.

Looking at the chronological development of car models for optimal control simulations, it can be noticed that car models complexity has continuously increased as consequence of the need for more accurate simulation outcomes. Model enhancements have been made possible thanks to the improved solving software, computer processing power, and modelling strategies. However, in most of the models that are being used till today (except in (9)) tyre loads are calculated in QSS conditions, as long as this allows to significantly reduce the numerical complexity of the resulting optimal control problem. Indeed in the QSS tyre load car models, the four suspensions dof together with three of the chassis dof (pitch, roll, and vertical displacement) can be neglected. A recent work (18) showed that the QSS tyre loads are a coarse approximation when using to optimise some car parameters. In such work, a car model endowed with suspensions (similar to the one in (9)) has been used for a full lap simulation on the Adria International Raceway circuit. The comparison between such multibody model and a QSS-based one showed that the QSS tyre loads assumption leads to different optimal values of the CoM position $(\approx 6 \mathrm{~cm})$ and suspension stiffness. Therefore, more complex vehicle models should be used for minimum time optimal control simulations to maximise accuracy. In particular tyre load transfer can be dynamically simulated if all the six chassis dof together with the four suspension-related dof are included in the car model. Compared to a QSS tyre loads model, this approach allows to simulate suspension related load transfer time delay, at the expense of seven additional dof. However the need for more accurate car models is limited by the solving capabilities of current software; when numerical complexity of the optimal control problem increases, it can take a long time to compute the solution, or even worse, the simulation may fail (19). Performing optimal control simulations with higher fidelity car models is not an easy task and it requires a proper formulation both of the car multibody model and of the optimal control problem.

State-of-the-art optimal control solving techniques mainly divide into indirect and direct methods. Indirect methods (20) (19) rely on the Pontryagin Maximum Principle to derive the first-order necessary conditions (differential equations) for the optimal control problem, which are then solved using common numerical techniques for differential equation systems. To the best knowledge of the authors, the most effective optimal control software for lap time simulations based on an indirect method is Pins, which has been used in $(15 ; 16)$. Differently, direct methods (20) (19) discretise first the optimal control problem so as to convert it to a Nonlinear Programming (NLP) problem, which is then solved using NLP software such as Ipopt, Knitro, Whorp, Snopt. Commercial software based on direct approach are Gpops, JModelica, Propt, Falcon, Psopt, Acado. Moreover, we recall that when dealing with optimal control problems, the well-known car models of commercial multibody software (like ADAMS, Virtual Lab Mo- 
tion, CarSim, RecurDyn, SimPack) present some set-backs. Indeed, numerical capabilities of solving any optimal control problem significantly increase when the exact Jacobian of the equations are provided $(19 ; 21 ; 20)$, and the analytic form of such expressions is generally required in order to calculate the Jacobian either analytically, or with automatic or complex differentiation techniques. Moreover, the analytic form allows fast and efficient evaluation of the equations, leading to a significant reduction in computing time.

In this work we present an optimal control based minimum time simulation of a GP2 car using a 14 dof car model. Particular attention has been put on the tyre load transfer dynamics and the QSS tyre loads assumption has been abandoned; moreover the full suspension kinematics are included in the model. The Pins software (22) has been chosen to solve the resulting optimal control problem since it is generally faster than NLP-based solvers (23). Pins requires the analytic expressions of the car model equations of motion which must be derived within the symbolic algebra software Maple. The analytic expressions of the equations are used by Pins to automatically generate both the first order necessary conditions and their Jacobian, which is a fundamental element for a fast and robust solution calculation.

In the next section the multibody model is described and the corresponding equations of motion are presented. The multibody model is composed by the main chassis (6 dof) and four wheels (4 spin dof), which are connected to the chassis by means of the suspension system (4 more dof). Suspension kinematics are accurately analysed in order to understand how tyre lateral and longitudinal forces contribute to determine the vehicle trim and thus load transfer. Then, the optimal control problem formulation is described and the minimum lap time simulation outcomes are presented. Simulation results are validated by comparison with experimental data acquired during a qualifying lap on the circuit of Montmelò in 2012.

\section{Multibody model of the GP2 Car}

As previously introduced, in this work the software used to solve the optimal-control minimum lap time problem is Pins, which requires the analytic expression of the equations of motion. In this section the car model is described and the corresponding equations of motion used to feed Pins are presented.

A GP2 car is a rear wheel drive formula car characterized by very stiff suspensions and high aerodynamic downforce generated by rear and front wings. The aerodynamics of GP2 cars is quite complex and wing force intensity depends on ride heights, therefore any model used to simulate GP2 car dynamics should carefully reproduce not only aerodynamic forces, but also all parameters that determine vehicle trim, first of all suspensions and tyres. The model here developed abandons the quasi-steady state tyre load simplification since it has been showed that it negatively influences simulations outcomes (18). Moreover, suspension kinematics are taken into consideration because they have a relevant influence on vehicle performance $(24 ; 25)$. A car model that includes this features leads to a relatively complex lap time optimal control problem, that may be very difficult to solve. The car mathematical model is thus a key element, together with the optimal control formulation and software used, to successfully solve the resulting problem.

The chassis is modelled as a rigid body free to translate and rotate about the three axes, as shown in figure 1, and SAE convention is adopted for the axes orientation. State variables associated to these six degrees of freedom (dof) are: the vehicle speed $V$, drift angle $\lambda$, centre of mass (CoM) vertical displacement $z$, yaw rate $\Omega_{z}$, pitch and roll angle $\mu$, $\phi$. Wheels are connected to the main chassis through short-long arm suspensions, that introduce one additional dof per wheel: the vertical motion of a generic wheel is named $z_{a s}$, where the suffix $a \in\{r, f\}$ stands for rear (r) or front (f) axle, while the suffix $s \in\{r, l\}$ stands for right (r) or left (l) side. As the suspension moves, the actual rigid motion of the wheel plane has been included in the model. Suspension forces take into account coil springs, torsion bars, dampers and anti-roll 
bars. Finally, wheel spins add four more dof $\omega_{a s}$. A torque sensitive differential is present at the rear axle. Tyre longitudinal forces are calculated through a nonlinear tyre model, and

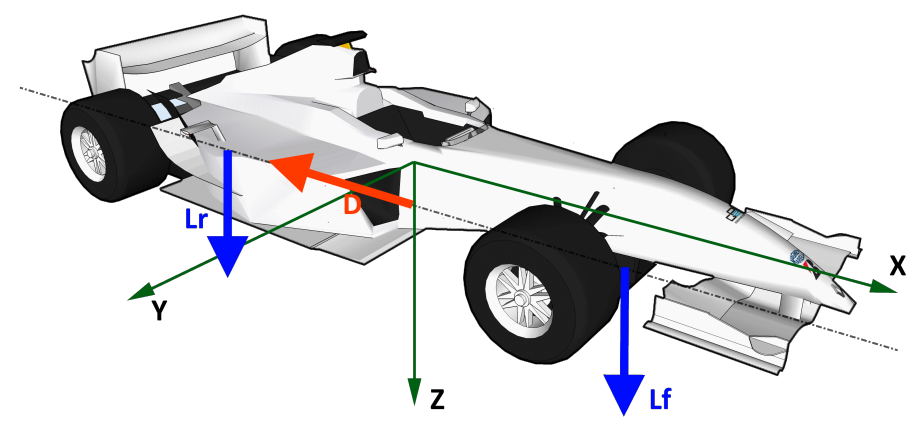

Figure 1: Car chassis degrees of freedom (dof)

aerodynamic drag and lift forces depend on ride heights. Summarizing, the model comprises 14 mechanical dof, which are reported in the table in appendix B.

\section{$2.1 \quad$ Equations of motion}

In this chapter the equations of motion will be derived. In a GP2 car, since the suspensions are very stiff, the chassis vertical displacement $z$, roll $\phi$ and pitch angles $\mu$ are very small (boundaries are $0 \mathrm{~mm}<z<25 \mathrm{~mm},|\phi|<1^{\circ},|\mu|<0.5^{\circ}$ ). The rigid motion of the chassis may be described by a sequence of the $4 \times 4$ Denavith Hartember transformation matrix $(26 ; 27)$ as follows:

$$
\boldsymbol{W}_{c}=\boldsymbol{W}_{c 0} \boldsymbol{T}(0,0, z-h) \boldsymbol{R}_{x}(\phi) \boldsymbol{R}_{y}(\mu)
$$

where $\boldsymbol{W}_{c 0}$ is a reference system that follows the longitudinal, lateral and yaw motion of the car along the circuit, $W_{c}$ is the chassis reference system and $h$ is the nominal distance of the CoM from ground. As the chassis pitch and roll angles can be considered small, every function of $\mu$ and $\phi$ has been approximated with its Taylor series expansion up to the first order. The resulting chassis reference system $\boldsymbol{W}_{c}$ therefore is:

$$
\boldsymbol{W}_{c}=\boldsymbol{W}_{c 0} \boldsymbol{T}(0,0, z-h) \boldsymbol{R}_{x}(\phi) \boldsymbol{R}_{y}(\mu)=\left(\begin{array}{cccc}
1 & 0 & \mu & 0 \\
0 & 1 & -\phi & 0 \\
-\mu & \phi & 1 & z-h \\
0 & 0 & 0 & 1
\end{array}\right)
$$

Moreover, also the time derivatives of $z, \phi$ and $\mu$ have been considered small, i.e. every function of $\dot{z}, \dot{\phi}, \dot{\mu}$ have been approximated with its Taylor series expansion up to the first order. Taking into account all the external forces acting on the vehicle (aerodynamic and tyre forces), it is possible to obtain Newton's equations of the full vehicle, these are:

$$
\begin{gathered}
m\left(\Omega_{z} V \lambda+\dot{V}\right)=\sum_{a s} S_{a s}-D-R-\psi_{f l} F_{f l}-\psi_{f r} F_{f r} \\
m\left(\Omega_{z} V-\dot{V} \lambda-V \dot{\lambda}\right)=\sum_{a s} F_{a s}+\psi_{f r} S_{f r}+\psi_{f l} S_{f l} \\
m(\ddot{z}-g)+\sum_{a s} m_{a s} \ddot{z}_{a s}=L_{f}+L_{r}-\sum_{a s} N_{a s}
\end{gathered}
$$

where $\Omega_{z}$ is the car yaw rate, $S_{a s}$ and $F_{a s}$ are the longitudinal and lateral tyre forces, $z$ is the car CoG coordinate along z-axis, $m_{a s}$ are wheel masses, $D$ is the drag force, $L_{f}, L_{r}$ are 
the aerodynamic downforce at the front and rear axles and $R$ is the rolling resistance of the four wheels. Again, the suffix $a \in\{r, f\}$ stands for rear (r) or front (f) axle, while the suffix $s \in\{r, l\}$ stands for right (r) or left (l) side; thus the suffix ' $a s$ ' can be one of the following: ' $r r$ ', ' $r l$ ', ' $f r$ ', ' $f l$ '. The acceleration terms related to the second order derivative of the wheel plate longitudinal $x_{a s}$ and lateral $y_{a s}$ displacements, as well as of the wheel plate camber $\phi_{a s}$, steering $\delta_{a s}$ and spin $\mu_{a s}$ angles, have been neglected, since they are small compared to the acceleration terms related to the chassis $(z, \phi, \mu)$ and wheel plate vertical displacement $z_{a s}$ (see appendix A). The Euler equations with respect to the point $\overrightarrow{\mathbf{A}}$, which is the origin of the reference system $\boldsymbol{W}_{c 0}$ defined before, are:

$$
\begin{array}{r}
I_{x x} \ddot{\phi}+\left(I_{z z} \mu-I_{x x} \mu-I_{x z}-\sum_{a s} m_{a s} b_{a s} z_{a s}\right) \dot{\Omega}_{z} \\
+\left(I_{z z}-I_{x x}-I_{y y}\right) \Omega_{z} \dot{\mu}-M\left(h V \dot{\lambda}+h \lambda \dot{V}+(z-h) \Omega_{z} V\right) \\
-\sum_{a s} m_{a s} t_{a s} \ddot{z}_{a s}-\left(\left(I_{y y}-I_{z z}\right) \phi+\sum_{a s} m_{a s} t_{a s} z_{a s}\right) \Omega_{z}^{2}-\sum_{a s} m_{a s} z_{a s} \Omega_{z} V \\
+\sum_{a s} I_{a s} \omega_{a s} \Omega_{z}=T_{x} \\
I_{y y} \ddot{\mu}+\left(\left(I_{y y}-I_{z z}\right) \phi+\sum_{a s} m_{a s} t_{a s} z_{a s}\right) \dot{\Omega}_{z}+\left(I_{x x}+I_{y y}-I_{z z}\right) \Omega_{z} \dot{\phi} \\
+M\left((z-h) \dot{V}-h \lambda \Omega_{z} V\right)+\sum_{a s} m_{a s} b_{a s} z_{a s}+\sum_{a s} m_{a s} z_{a s} \dot{V} \\
+\left(\left(I_{z z}-I_{x x}\right) \mu-I_{x z}+\sum_{a s} m_{a s} b_{a s} z_{a s}\right) \Omega_{z}^{2}=T_{y} \\
\left(I_{z z}+2 I_{x z} \mu\right) \dot{\Omega}_{z}-\sum_{a s} I_{a s} \omega_{a s} \dot{\phi}-I_{x z}\left(\ddot{\phi}-2 \Omega_{z} \dot{\mu}\right)=T_{z}
\end{array}
$$

where $I_{x x}, I_{y y}, I_{z z}$ are the principal moments of inertia of the car chassis, $I_{x z}$ is the cross moment of inertia, $I_{r r}=I_{r l}=I_{r}$ are the rear wheel spin inertia moment, $I_{f r}=I_{f l}=I_{f}$ are front wheel spin inertia moment, $t_{r}=t_{r l}=-t_{r r}$ are the rear half track, $t_{f}=t_{f l}=-t_{f r}$ is the front half track, $b_{r}=b_{r r}=b_{r l}$ is the $\mathrm{x}$-axis distance of the car CoM from the rear axle, and $b_{f}=-b_{f r}=-b_{f l}$ is the x-axis distance of the car CoM from the front axle. $T_{x}, T_{y}$, and $T_{z}$ are respectively the $\mathrm{x}, \mathrm{y}$ and $\mathrm{z}$ component of the net external torque acting on the chassis:

$$
\begin{gathered}
T_{x}=\sum_{a s} N_{a s}\left(t_{a s}+h \phi-y_{a s}+r_{a s} \gamma_{a s}\right) \\
T_{y}=L_{r} b_{r}-L_{f} b_{f}+\sum_{a s} N_{a s}\left(-b_{a s}+\left(h-r_{a s}\right) \mu+x_{a s}\right) \\
T_{z}=S_{f r} b_{f} \delta_{f r}+S_{f l} b_{f} \delta_{f l}+\sum_{a s} S_{a s}\left(t_{a s}+h \phi-y_{a s}+r_{a s} \gamma_{a s}\right)+ \\
-F_{f l} t_{f} \delta_{f l}+F_{f r} t_{f} \delta_{f r}-\sum_{a s} F_{a s}\left(b_{a s}-\left(h-r_{a s}\right) \mu-x_{a s}\right)
\end{gathered}
$$

where $r_{r r}=r_{r l}=r_{r}$ are the rear tyre radius, and $r_{f r}=r_{f l}=r_{f}$ is the front tyre radius.

The equations of motion governing the suspension dof (i.e. wheel plate vertical displacement) are obtained using the Lagrangian approach which, in contrast to the Newton one, allows the suspension links reaction forces to be disregarded. With the generalized force approach, the suspension equations can be derived from:

$$
\frac{d}{d t}\left(\frac{\partial K_{a s}}{\partial \dot{z}_{a s}}\right)-\frac{\partial V_{a s}}{\partial z_{a s}}=Q_{a s}
$$


where $\dot{z}_{a s}$ is the time derivative of the wheel vertical displacement $z_{a s}, K_{a s}$ is the kinetic energy of the wheel, $V_{a s}$ is the gravitational potential energy, and $Q_{a s}$ is the generalized force acting on the wheel. Suspension equations take into account for the suspension kinematics, i.e. that tyre contact point moves fore and aft as well as left and right in the road plane as the suspension moves up and down, so as that tyre lateral and longitudinal forces influence the equilibrium of the suspension. Moreover, each wheel not only translates as the suspension moves, but it also rotates about all the three axes; such rotations influence the in-plane forces generated by the tyre as long as they modify both the tyre sideslip and camber angles. Then the explicit equation form is:

$$
\begin{array}{r}
m_{a s}\left(\ddot{z}-\ddot{z}_{a s}+b_{a s} \ddot{\mu}-t_{a s} \ddot{\phi}-g\right)=-J_{a s}-N_{a s} \\
+S_{a s}\left(\frac{\partial x_{a s}}{\partial z_{a s}}+\frac{\partial \mu_{a s}}{\partial z_{a s}} r_{a s}+\frac{\partial y_{a s}}{\partial z_{a s}} \psi_{a s}+\frac{\partial \psi_{a s}}{\partial z_{a s}} \phi_{a s} r_{a s}\right) \\
-F_{a s}\left(\frac{\partial y_{a s}}{\partial z_{a s}}+\frac{\partial \phi_{a s}}{\partial z_{a s}} r_{a s}+\frac{\partial x_{a s}}{\partial z_{a s}} \psi_{a s}\right)
\end{array}
$$

where $m_{a s}$ is the wheel mass, $r_{a s}$ is the tyre radius, $x_{a s}, y_{a s}$ are respectively the wheel plate longitudinal and lateral displacements, $\phi_{a s}, \psi_{a s}, \mu_{a s}$ are respectively the wheel plate camber steering and spin angles, and $J_{a s}$ is the suspension force acting on the chassis and counterreacting on the wheel. The terms $\partial x_{a s} / \partial z_{a s}, \partial y_{a s} / \partial z_{a s}, \partial \mu_{a s} / \partial z_{a s}, \partial \gamma_{a s} / \partial z_{a s}$ are those related to suspension kinematics and, as it can be noticed, they determine how the tyre longitudinal and lateral forces contribute to suspension motion. The detailed analysis of the suspensions kinematics is provided in appendix A. When equation (7) is derived from (6), the terms related to the time derivative of the wheel plate variables in $\mathbf{u}_{a s}=\left\{x_{a s}, y_{a s}, \phi_{a s}, \psi_{a s}, \mu_{a s}\right\}$ have been neglected as long as they do not have a relevant physical effect on suspension dynamics. Indeed such terms are related to small inertial forces (less than 0.1 times the inertial force related to $\ddot{z}_{a s}$ ) that do not play an important role in time simulations (18). On the contrary, the terms related to the ratio between variables in $\mathbf{u}_{a s}$ and $z_{a s}$ are required to take into account the antilift, anti-squat or scrub behaviour of the suspension and are relevant even in determining the stationary trim of the suspension or of the vehicle $(24 ; 25)$.

Wheel spin motion is governed by tyre forces and the driver's braking or driving input torques. Euler equations for the wheel spins are:

$$
I_{a s} \dot{\omega}_{a s}=T_{a s}-S_{a s}\left(r_{a s}-z_{a s}-z-b_{a s} \mu+t_{a s} \phi\right)
$$

where $I_{a s}$ is wheel inertia moment around the spin axis (including also half of the axle and powertrain inertias in the rear wheels), $r_{a s}$ is the tyre radius, $S_{a s}$ is the tyre longitudinal force and $T_{a s}$ is the torque delivered to the wheel. In the above equation the inertial terms related to the fact that the wheel spin axis is not fixed but moves with and with respect the chassis have been neglected because their expression is complex but their effect is negligible.

\section{$2.2 \quad$ Forces}

The multibody car model here presented is fully described by the chassis Newton (3) and Euler (4) equations, together with the suspension (7) and wheel spin (8) ones, however the forces that appears in these equations still have to be made explicit. In this section the suspension, tyre and aerodynamic forces, together with the wheel driving torques are expressed in terms of the model state variables and controls.

The four torque inputs $T_{a s}$ depend on one variable, the overall driving torque $T$, which is the sum of the engine (positive part, $T_{e}$ ) and braking (negative part, $T_{b}$ ) torque; moreover the simultaneous presence of a driving torque at the rear axle and a braking torque at the front one is not allowed:

$$
T=f^{+}(T)+f^{-}(T) \equiv T_{e}+T_{b}
$$


where $f^{-}$and $f^{+}$return respectively the (regularized) negative and positive part of the argument. It can be noticed that the engine torque is only positive, in other words we are neglecting the engine brake torque; this is a consequence of the lack of experimental data regarding the negative torque exerted by the engine at zero throttle. The traction torque $T_{e}$ is delivered only to the rear axle, while the braking one $T_{b}$ is split between both axes with a constant front braking bias $\beta$. Moreover, at the rear axle a torque sensitive differential is present. Thus, the torque delivered to each wheel is:

$$
\begin{gathered}
T_{r r}=\frac{T_{e}}{2}+T_{e} k_{d}\left(\omega_{r l}-\omega_{r r}\right)+(1-\beta) \frac{T_{b}}{2} \operatorname{sign}\left(\omega_{r r}\right) \\
T_{r l}=\frac{T_{e}}{2}-T_{e} k_{d}\left(\omega_{r l}-\omega_{r r}\right)+(1-\beta) \frac{T_{b}}{2} \operatorname{sign}\left(\omega_{r l}\right) \\
T_{f r}=\beta \frac{T_{b}}{2} \operatorname{sign}\left(\omega_{f r}\right) \\
T_{f l}=\beta \frac{T_{b}}{2} \operatorname{sign}\left(\omega_{f f}\right)
\end{gathered}
$$

where $k_{d}$ is the differential stiffness and sign is the (regularized) signum function which has been introduced to cut the braking torque applied to the wheel when it locks, so as to prevent a non-physical backward wheel spin. Suspensions forces $J_{a s}$ acting on the chassis and counterreacting on each wheel are the sum of elastic (spring), damper, anti-roll bar $J_{a s}^{a}$ and bump rubber $J_{a s}^{p}$ forces:

$$
J_{a s}=K_{a s}^{s} z_{a s}+K_{a s}^{d} \dot{z}_{a s}+J_{a s}^{a}+J_{a s}^{p}
$$

where $K_{a s}^{s}$ is the elastic force stiffness exerted by the torsion bars (front suspensions) or coil springs (rear suspensions), $K_{a s}^{d}$ is the damping coefficient and $z_{a s}$ is the wheel travel. The elastic and damping forces are expressed by a linear relationship as long as the velocity ratio between the wheel travel and torsion bar rotation angle or damper travel is constant in the working range of the suspensions. The anti-roll bar forces $J_{a s}^{a}$ depend on the difference between right and left wheel displacement:

$$
\begin{gathered}
J_{r r}^{a}=K_{r}^{a}\left(z_{r r}-z_{r l}\right)=-J_{r l}^{a} \\
J_{f r}^{a}=K_{f}^{a}\left(z_{f r}-z_{f l}\right)=-J_{f l}^{a}
\end{gathered}
$$

where $K_{r}^{a}$ and $K_{f}^{a}$ are the rear and front reduced anti-roll bar stiffness. The last force $J_{a s}^{p}$ in equation (11) is the force due to the bump rubbers, which prevent the suspensions from excessive travel. The bump rubber forces, which are comparable or greater than the coil spring or torsion bar ones even for wheel vertical travels of few millimeters and makes the suspension rates highly highly non-linear, are given by splines used to fit the experimental force vs deflection curve data. In a GP2/F1 car model, tyres cannot be considered completely rigid as long as their radial stiffness is comparable to that of suspensions. The tyre manufacturer provides a specific formula (28) to calculate the stationary tyre radial deformation as function of the tyre load, spin, pressure, camber and lateral force:

$$
\xi_{a s}=\frac{N_{a s}}{p_{1} P_{a s}+a_{1} \omega_{a s}^{2}+a_{2} \omega_{a s}+a_{3}+c_{1}\left|\gamma_{a s}\right|+f_{1} F_{a s}^{2} / N_{a s}}+b_{1} \omega_{a s}^{2}+b_{2} \omega_{a s}
$$

where $N_{a s}$ is the tyre load, $\xi_{a s}$ the tyre radial deformation, $\omega_{a s}$ the wheel spin, $P_{a s}$ is the tyre pressure, $p_{1}, a_{1}, a_{2}, a_{3}, c_{1}, f_{1}, b_{1}$ and $b_{2}$ are constant coefficients. As long as tyre radial deformations are determined by the chassis and suspensions trim, while tyre loads are not, we numerically inverted the previous formula in order to obtain the tyre loads as function of the other variables:

$$
N_{a s}=f^{+}\left(n_{d a} \xi_{a s}+n_{d o a} \xi_{a s} \omega_{a s}+n_{o a} \omega_{a s}^{2}+n_{y a} F_{a s}^{2}+n_{c a} \dot{\xi}\right)
$$


where $n_{d a}, n_{d o a}, n_{o a}$ and $n_{y a}$ are the coefficients used to fit the data calculated using (13). The camber dependence has been neglected, and the pressure has been considered to be constant at $19 p s i$ for the rear tyre, and 20psi for the front one. A damping coefficient $n_{c}$ has been added to take transient behaviour into account. The $f^{+}$function ensures that the tyre load never becomes negative. As previously pointed out, the tyre radial deformations are determined by the chassis and suspension trims and are given by the following relationship:

$$
\xi_{a s}=z+z_{a s}+\mu b_{a s}-\frac{1}{2} \phi t_{a s}
$$

where $t_{r}=t_{r l}=-t_{r r}$ is the rear half track, $t_{f}=t_{f l}=-t_{f r}$ is the front half track, $b_{r}=b_{r r}=b_{r l}$ is the $\mathrm{x}$-axis distance of the car CoM from the rear axle, and $b_{f}=-b_{f r}=-b_{f l}$ is the $\mathrm{x}$-axis distance of the car CoM from the front axle.

With the approach we have adopted, lateral and longitudinal load transfers are automatically calculated according to the model state variables as well as suspension characteristics. Longitudinal $S_{a s}$ and lateral $F_{a s}$ tyre forces are given by a Magic Formula Tyre Model 5.1 (17), as functions of tyre load, longitudinal slip, sideslip and camber angles. Moreover a relaxation equation for every tyre force has been added because it is known that tyre forces raise with a certain time delay with respect to input variables, especially in the case of lateral forces (17). Thus, the equations governing the tyre force relaxations are:

$$
\begin{aligned}
& \frac{\sigma_{x}}{V_{a s}} \dot{S}_{a s}+S_{a s}=S_{m a g i c}\left(N_{a s}, \kappa_{a s}, \lambda_{a s}, \phi_{a s}\right) \\
& \frac{\sigma_{y}}{V_{a s}} \dot{F}_{a s}+F_{a s}=F_{m a g i c}\left(N_{a s}, \kappa_{a s}, \lambda_{a s}, \phi_{a s}\right)
\end{aligned}
$$

where $\phi_{a s}$ is the wheel camber angle, $\sigma_{x}$ and $\sigma_{y}$ are the relaxation lengths of the tyre in the longitudinal and lateral direction, $S_{\text {magic }}$ and $F_{\text {magic }}$ are the stationary Magic Formula tyre forces, $\kappa_{a s}, \lambda_{a s}, \phi_{a s}$ are respectively the tyre longitudinal slip, sideslip angle and camber angle. The slip quantities necessary to calculate the tyre forces can be obtained as function of the model state variables as shown below in (17). Only the expressions for the rear right tyre are shown in (17) as long as similar ones apply to the other tyres.

$$
\begin{gathered}
V_{x, r r}=V-\Omega t_{r}+\dot{\mu}\left(h-r_{r 0}\right)+\dot{x}_{r r}+\Omega \phi h \\
+\Omega \phi_{r r} r_{r 0}-\Omega b\left(\psi_{r r}+\psi_{r 0}\right)-\Omega y_{r r} \\
V_{y, r r}=\Omega\left(x_{r r}-b\right)-\dot{\phi} h-\dot{\gamma}_{r r} r_{r 0}+\dot{y}_{r r}-V \lambda \\
+\left(h-r_{r 0}\right) \Omega \mu+\left(\Omega t_{r}-V\right)\left(\psi_{r r}+\psi_{r 0}\right) \\
V_{r, r r}=\dot{\mu}_{r r} r_{r 0}-\omega_{r r}\left(r_{r 0}+z+z_{r r}+b \mu+t_{r} \phi\right) \\
\kappa_{r r}=\frac{V_{r, r r}}{V_{x, r r}}-1 \\
\lambda_{r r}=\frac{V_{y, r r}}{V_{x, r r}} \\
\phi_{r r}=\phi+\gamma_{r r 0}+\gamma_{r r} \\
\psi_{r r}=\delta_{r r}+\delta_{r r 0}
\end{gathered}
$$

where $\phi_{r r}$ and $\psi_{r r}$ are respectively the rear right wheel camber and steer angle, $\gamma_{r r 0}, \delta_{r r 0}$ are the rear right wheel static camber and steer angle; moreover in the computation of $\lambda_{r r}$ the approximation $\tan (x) \approx x$ holds.

The rolling resistance of each tyre is proportional to the tyre load. Thus, the total rolling resistance force has been approximated with the following expression:

$$
R=\rho_{r}\left(M g \frac{b_{f}}{w}+L_{r}\right)+\rho_{f}\left(M g \frac{b_{r}}{w}+L_{f}\right)
$$


where $\rho_{r}$ and $\rho_{f}$ are the rear and front tyre rolling resistance coefficients.

Aerodynamic forces that act on the car are as follows:

$$
\begin{aligned}
D & =\frac{1}{2} \rho C_{d}\left(h_{r}, h_{f}\right) V^{2} \\
L_{r} & =-\frac{1}{2} \rho C_{l r}\left(h_{r}, h_{f}\right) V^{2} \\
L_{f} & =-\frac{1}{2} \rho C_{l f}\left(h_{r}, h_{f}\right) V^{2}
\end{aligned}
$$

where $D$ is the drag force, $L_{r}$ and $L_{f}$ are respectively the rear and front aerodynamic downforce, $\rho$ is air density, $C_{d}, C_{l r}, C_{l f}$ are the drag, rear lift and front lift coefficients that depend on the rear $h_{r}$ and front $h_{f}$ ride heights as third-degree polynomials. The coefficients used in such polynomials are those provided by the car manufacturer. The average front and rear ride heights can be easily calculated as function of $z$ and $\mu$ on the basis of elementary geometrical considerations:

$$
\begin{gathered}
h_{r}=h_{r 0}-\mu b_{r}+z \\
h_{f}=h_{f 0}+\mu b_{f}+z
\end{gathered}
$$

where $h_{r 0}$ and $h_{f 0}$ are respectively the rear and front ride height nominal values, $b_{r}$ and $b_{f}$ are the $\mathrm{x}$-axis distance of the rear and front axle from the CoM.

\section{Optimal Control Problem}

\subsection{Road and vehicle tracking}

In minimum time simulations a road model is required. The approach used to describe both the track and the vehicle position along the circuit is the same described in other works like (29), so we are not going to describe it deeply; the reader can refer to that article for more details. The road is described by means of the line curvature $\kappa$ as a function of the road centre line curvilinear abscissa $s$ (see figure 2); the $(x, y)$ position of the road and its heading can then be obtained by simple integration (29). The car position and orientation is instead described by three variables: the curvilinear abscissa along the road centre line $s$, the lateral displacement from the centre line $n$ and the relative heading to road centre line direction $\alpha$, as shown in figure 2. The equations governing the time evolution of the tracking coordinates are:

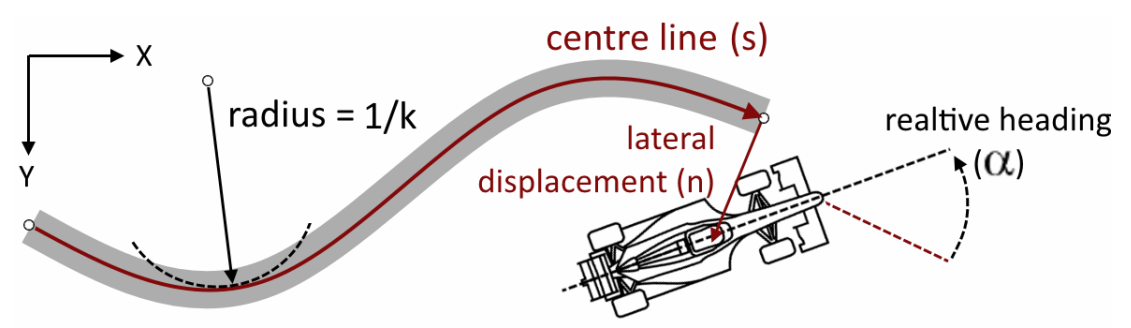

Figure 2: Road tracking: the curvilinear abscissa $s$, car lateral displacement from road centre line $n$ and car relative heading angle $\alpha$ are displayed.

$$
\begin{gathered}
\dot{s}=\frac{V(\lambda \sin \alpha+\cos \alpha)}{1-n \kappa(s)} \\
\dot{n}=V \sin \alpha-V \lambda \cos \alpha \\
\dot{\alpha}=\Omega_{z}-\kappa(s) \frac{V \cos \alpha+V \lambda \sin \alpha}{1-n \kappa(s)}
\end{gathered}
$$


Equations (26) track the vehicle position and orientation relative to the road by simply integrating the vehicle speed and drift angle together with road curvature, so the vehicle lateral position $n$ and relative heading $\alpha$ are immediately available as state variables. This is a remarkable advantage in comparison to the Cartesian coordinate approach, as described in (29).

\subsection{State space formulation}

The optimal control problem solution strategy used in this work (as many others) requires the model dynamics to be describe by a set of first-order ordinary differential equations (ODE). Newton's (3), Euler's (4) and suspension (7) equations may be immediately reduced to an ODE system by introducing auxiliary variables for the relevant chassis and suspension speeds:

$$
\begin{aligned}
\dot{z} & =v \\
\dot{\phi} & =\Omega_{x} \\
\dot{\mu} & =\Omega_{y} \\
\dot{z}_{a s} & =v_{a s}
\end{aligned}
$$

At this point, equations (3) to (22) completely describe car dynamics as a system of 32 first order differential equations with as many state variables and 2 inputs, respectively the overall driving torque $T$ and the steering rack displacement $y_{r}$. However, optimal control solutions typically include abrupt variation of the control inputs, which conflict with the dislike of jerk felt by human drivers $(30 ; 31 ; 32)$. To obtain smoother manoeuvres, the model is therefore controlled by the steering angle rate and the longitudinal jerk. According to this, the two driver inputs $T$ and $y_{r}$ have been included to the state variables, while the controls have been moved to their time derivatives, $j_{u}$ and $j_{y}$, satisfying the following equations:

$$
\begin{aligned}
& j_{y}=\dot{y}_{r} \\
& j_{u}=\dot{T}
\end{aligned}
$$

where $j_{u}$ is the longitudinal jerk control, which (mainly) controls the longitudinal dynamics, and $j_{y}$ is the lateral jerk control, which (mainly) controls the lateral dynamics.

In conclusion, vehicle dynamics is described by means of a set of 34 state variables:

$$
\boldsymbol{x}=\left\{s, n, \alpha, V, \lambda, z, v, \phi, \Omega_{x}, \mu, \Omega_{y}, \Omega_{z}, z_{a s}, v_{a s}, \omega_{a s}, F_{a s}, S_{a s}, y_{r}, T\right\}^{T}
$$

plus two inputs:

$$
\boldsymbol{u}=\left\{j_{y}, j_{u}\right\}^{T}
$$

and as many implicit first order differential equations, which may be abbreviated to:

$$
A(x) \dot{x}=f(x, u)
$$

where matrix $\boldsymbol{A}$ is invertible provided that $V>0$, i.e. the vehicle never stops, and $n<1 / \kappa(s)$, i.e. the vehicle never passes over the local curvature centre of the road.

\subsection{Optimal Control Problem formulation}

The minimum time problem is here formulated as an indirect optimal control problem with the same approach described in previous works (18) and (33), thus it will be described only briefly; the reader can refer to those works for further information. The Optimal Control Problem (OCP) allows to find the vehicle control inputs $\boldsymbol{u}=\left\{j_{u}, j_{y}\right\}^{T}$ that drive the vehicle from the starting grid line $s=0$ and $t(0)=0$ to the finish one $s=L$ in the minimum time $T=t(L)$, while 
satisfying a set of both equality and inequality constraints. The OCP formulation accepted by the software used, Pins, is the following:

$$
\begin{aligned}
\text { find: } & \min _{\boldsymbol{u} \in \boldsymbol{U}} T \\
\text { subject to: } & \boldsymbol{A} \dot{\boldsymbol{x}}=\boldsymbol{f}(\boldsymbol{x}, \boldsymbol{u}, t) \\
& \boldsymbol{\psi}(\boldsymbol{x}, \boldsymbol{u}, t) \leq \mathbf{0} \\
& \boldsymbol{b}(\boldsymbol{x}(0), \boldsymbol{x}(T))=\mathbf{0}
\end{aligned}
$$

where $\boldsymbol{x}$ is the state variable vector and $\boldsymbol{u}$ is the input one (24), (27b) is the state space model (i.e. the equations of motion (26)), (27d) is the set of boundary conditions used to specify the vehicle state at the beginning and at the end of the manoeuvre and (27c) is a set of algebraic inequalities that may bound both the state variables and control inputs. In the specific case of our car model, equations (27c) are used to make the simulation withstand to real physical constraints; the first one ensures that the car position remains always within the track borders:

$$
-\left(w_{l}-t_{w} \cos (\alpha)\right) \leq n \leq w_{r}-t_{w} \cos (\alpha)
$$

where $n$ is the lateral displacement of the vehicle from the road centre, $t_{w}$ is the overall car width, $w_{r}$ and $w_{l}$ are the right and left half-road widths which might vary along the track. Such constraint is a good approximation of the real car borders limit thanks to the limited sideslip angle of the car. Moreover, a second inequality has been added into (27c) so as to ensure that the driving torque delivered to the wheels does not exceed that which the engine can deliver $T_{e, \max }$ :

$$
T_{e} \leq \tau_{e} T_{e, \max }\left(\tau_{e}\left(\omega_{r r}+\omega_{r l}\right)\right)
$$

where $\tau_{e}$ is the transmission ratio between the rear axle and the engine spin (which depends on the gear engaged); clearly, the maximum available engine torque $T_{e, \max }$ depends on the engine speed. A third constraint has been added to the optimal control formulation so as to prevent the ideal driver from locking the front wheels when braking: indeed real drivers tend to avoid such manoeuvre as long as it causes high tyre wear and makes the tyre to loose performance. Mathematically this constraint has been expressed as:

$$
\begin{aligned}
& \kappa_{f r} \geq \kappa_{\text {min }} \\
& \kappa_{f l} \geq \kappa_{\text {min }}
\end{aligned}
$$

where $\kappa_{\min } \approx-0.6$ is the minimum slip value as it would result in unacceptable tyre wear. Finally, the control inputs $j_{u}, j_{y}$, which are strictly related to the driving torque and steering angle rate by equations (23), are limited in magnitude so as to avoid control rates higher than what a human driver can sustain:

$$
\begin{aligned}
-j_{u, \max } & \leq j_{u} \leq j_{u, \max } \\
-j_{y, \max } & \leq j_{y} \leq j_{y, \max }
\end{aligned}
$$

where $j_{u, \max }$ and $j_{y, \max }$ are the maximum value allowed for $j_{u}$ and $j_{y}$ controls.

With the indirect optimal control method used in this work the inequality constraints (28) (29) (31) are converted into penalty terms that are added to the problem target in (27a). The optimisation problem is thus reduced to a constrained minimisation problem, where the constraints are the first-order equations (27b). The controls that minimise the target are then found by solving the first-order necessary conditions (differential equations) that can be obtained through the calculus of variations or the Pontryagin Minimum Principle. The software used, Pins, automatically converts inequality constraints into penalty terms and derives the firstorder necessary conditions. Pins is composed by a Maple package (called XOptima) for the 
code generation of the first-order necessary conditions, an embedded ruby interpreter (called Pins) to specify the problem numerical data, and a $C++$ library (called Mechatronix) that solves the problem. A detailed description of the underlying algorithm of the Pins solver can be found in (34).

\subsection{Lap time simulation and model validation}

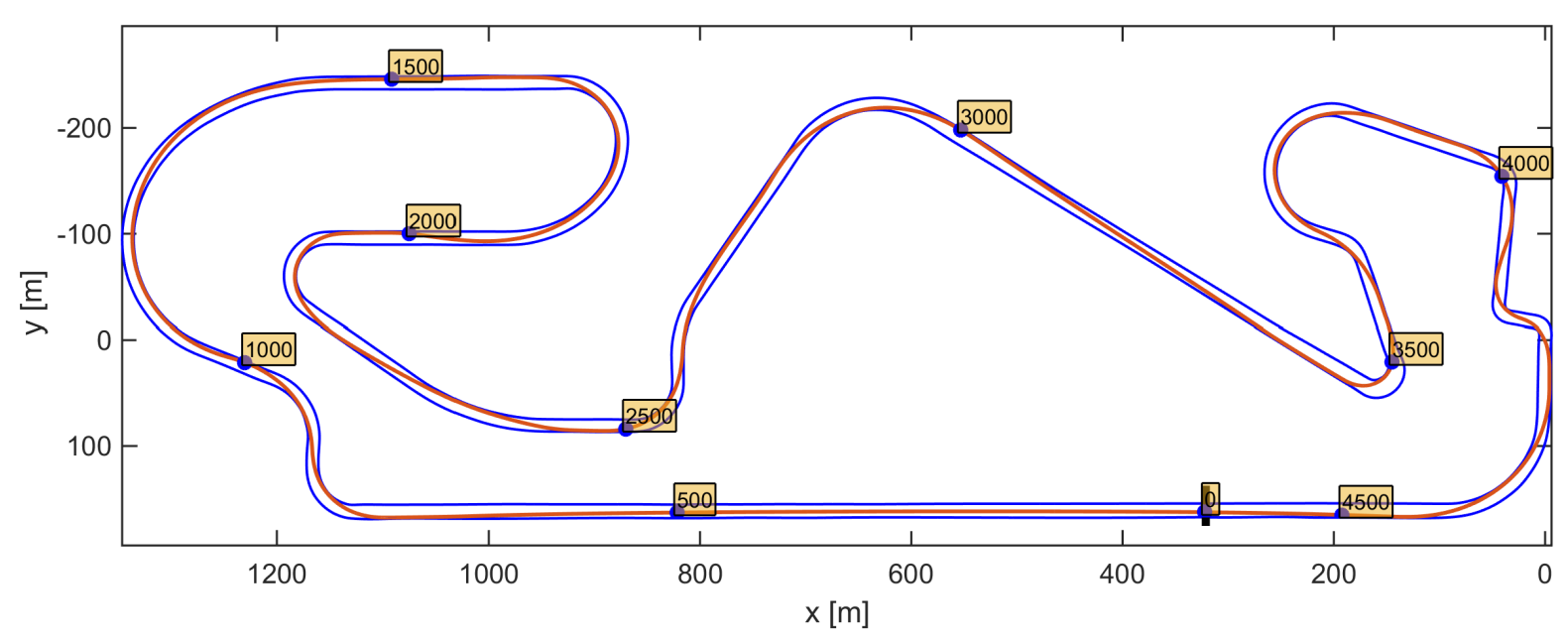

Figure 3: Optimal trajectory on Montmelò circuit. Numbers within ochre boxes indicate the value of the road centre line abscissa in meters.

Optimal control simulation has been carried out on the circuit of Montmelò in Barcelona. The simulation took approximately 26 minutes to be computed on an Intel Core it based desktop computer, suggesting the proposed model, together with the used software, is very efficient. In the first part of this section the car model will be validated through a comparison between simulation results and the experimental data acquired in a qualifying lap of a driver in 2012 GP2 season, while the second part will be focused in the analysis of the car dynamics.

Figure 3 shows the car trajectory resulting from the simulation, where the road geometry has been reconstructed by getting GPS road coordinates from Google satellite photos. As expected, the ideal driver tends to smooth the trajectory through corners in order to achieve the minimum time. Figure 4 compares the simulated and the experimental speed profiles; there is good agreement between simulation and telemetry especially in the first two thirds of the track, while in the last sector the simulated speed is slightly higher than the real one in correspondence of the corner apex point. Indeed the simulated lap time $(91.287 \mathrm{~s})$ is lower than the real one (91.600s) by approximately $0.4 \mathrm{~s}$. It is known that in a qualifying lap at the Montmelò circuit, in the third sector rear tyres are very warm and their performance tends to decrease, thus this phenomenon might generate the discrepancies between simulated and real speed profile that arises in that part of the track. In figure 4 both longitudinal $a_{x}$ and lateral $a_{y}$ accelerations are also reported, and it can be observed that the simulated ones are close to the real ones; $a_{x}$ and $a_{y}$ bounds are of approximately $-40 \mathrm{~m} / \mathrm{s}^{2}<a_{x}<20 \mathrm{~m} / \mathrm{s}^{2}$ and $\left|a_{y}\right|<30 \mathrm{~m} / \mathrm{s}^{2}$. The resulting g-g diagram, which is represented in the bottom-left corner of figure 5 , highlights an ellipsoidal shape for positive accelerations, while it has a remarkable triangular shape for negative ones; this difference arises mainly because of the front inner wheel locking that occurs when braking while steering, limiting the lateral acceleration. The maximum accelerations that the car can withstand highly depend on the speed, as long as the aerodynamic downforce increases with the square of the speed. The influence of the speed on the acceleration limits is also shown in figure 5: $a_{x}$ is the one which varies more with the speed, passing from $-20 \mathrm{~m} / \mathrm{s}^{2}<a_{x}<20 \mathrm{~m} / \mathrm{s}^{2}$ at a 
speed of $\approx 100 \mathrm{~km} / \mathrm{h}$ up to $-40 \mathrm{~m} / \mathrm{s}^{2}<a_{x}<5 \mathrm{~m} / \mathrm{s}^{2}$ at a speed of $\approx 250 \mathrm{~km} / \mathrm{h}$, while the lateral acceleration increases only from $-20 \mathrm{~m} / \mathrm{s}^{2}<a_{y}<20 \mathrm{~m} / \mathrm{s}^{2}$ to $-28 \mathrm{~m} / \mathrm{s}^{2}<a_{y}<28 \mathrm{~m} / \mathrm{s}^{2}$ for the same speed range. From speed and acceleration comparisons, we can state that the model developed in this work is able to well reproduce the dynamics of a GP2 car. Some differences are clearly present, but they could be reduced with a better measurement of some parameters affecting the performance, first of all tyre characterisation and road geometry.

The motion of the chassis along the track is shown in figure 6 , where the vertical displacement $z$, together with roll $\phi$ and pitch $\mu$ angles are reported. All these quantities are small, indeed $z$ is comprised between $0 \mathrm{~mm}$ and $25 \mathrm{~mm}$, the roll angle is lower than $1^{\circ}$ and the pitch angle is never larger than $0.5^{\circ}$. The very limited chassis displacements are consequence of the high suspension stiffness, which is even greater than that of the tyres due to the presence of suspension bump rubbers. Indeed, suspension travels are in the range $-10 \mathrm{~mm}<z_{\text {as }}<7 \mathrm{~mm}$ (where negative values correspond to a compressed suspension), while tyre radial deformation spans in $0 \mathrm{~mm}<\xi_{a s}<30 \mathrm{~mm}$, as it can be observed in figure 7 . Within such limited range of suspension travel and tyre deformation, tyre loads vary from almost 0 to $6000 \mathrm{~N}$. Even if chassis and suspensions motions are so limited, they noticeably affect both aerodynamic forces and load transfers. Figure 6 shows also the aerodynamic drag and downforce together with the ride heights and the downforce balance (i.e. the front by total downforce ratio $L_{f} /\left(L_{f}+L_{r}\right)$ ). In such figure, the aerodynamic force trends resemble that of the speed, due to the strict dependence of the former on the square of the latter. However, as long as ride heights change along the track, varying in the range $-5 \mathrm{~mm}<h_{f}<21 \mathrm{~mm}$ (front ride height) and $40<h_{r}<60$ (rear ride height), the downforce balance also changes. Indeed the downforce balance generally increases with the speed, moreover it bumps up in correspondence of high braking manoeuvres. It can be observed that, when the car withstands high negative accelerations, the front ride height decreases while the rear one increases due to the chassis pitch, thus affecting aero balance. Moreover, the minimum value of the front ride height is negative $(-5 \mathrm{~mm})$ which might seem non-realistic; however, it should be considered that the leading edge of the skid plane is located well behind the front axle (that is where the front ride height is calculated), and that the skid plane is quite flexible, thus slightly negative values for the front ride heights are very likely to be reached when the car is bottoming on the road surface.

As previously said, chassis and suspension motions not only influence aerodynamic forces, but also load transfers. The lateral load transfer at the rear axle $\left(N_{r}^{l a t} \equiv N_{r r}-N_{r l}\right)$ versus that at the front one $\left(N_{f}^{l a t} \equiv N_{f r}-N_{f l}\right)$ resulting from the simulation is shown in figure 8, where the two dashed lines correspond to a constant ratio $N_{r}^{\text {lat }}=1.12 N_{f}^{\text {lat }}$ and $N_{r}^{\text {lat }}=0.66 N_{f}^{\text {lat }}$. It is clear that the roll balance ratio $N_{r}^{\text {lat }} / N_{f}^{\text {lat }}$ changes by a factor of almost 2 along the track, depending on the trim of the car. Since the roll balance ratio is determined, at a first approximation, by the ratios between tyre, anti-roll bar, coil spring and torsion bar stiffness, its variation is caused by the highly non-linear suspension rates (the non-linearity is due to the bump rubber forces). In different words, depending on the instant trim of the car, each wheel "sees" a different reduced vertical stiffness to the chassis, and the roll balance ratio varies as a consequence. This effect instead is not captured by the most commonly used QSS car models where, in order to determine the loads on each wheel, it is generally assumed that the roll balance ratio is constant. Indeed this is what is done in works as $(6 ; 7 ; 8 ; 14 ; 10 ; 11)$ and $(12)$.

\section{Conclusions}

The state of the art of minimum time optimal control simulations for race cars has been considerably improved in the last years and relatively complex car models can nowadays be used for such simulations. However, car multibody models for optimal control simulations are still generally based on quasi steady state tyre loads in order to reduce the numerical size of the resulting problem and to make the problem easier to solve. Such assumptions can lead to suboptimal 
results when lap time simulations are used to optimize car setup parameters; this paper fills this gap by proposing an accurate car model which includes wheels and suspension dynamics (thus, load transfer dynamics too) but it is concise enough for optimal control applications thanks to the symbolic approach. More in detail, we have developed a GP2 formula car multibody model for optimal control simulations which include: chassis, suspension and wheel dynamics, full Magic Formula tyre forces, non linear tyre loads and ride-height-dependent aerodynamic forces. Suspension kinematics have been deeply analysed and included in the car model, as long as it has a significant effect on tyre loads; moreover their impact on overall car performance has been highlighted in steady state conditions. An indirect optimal control approach has then been adopted to successfully perform a full lap time simulation on the circuit of Montmelo', and the model has been validated by comparison with the telemetry data of an official qualifying lap in 2012. The simulation outcomes and the experimental data have shown a good agreement in speed, accelerations and accelerations-speed dependence. Moreover, the simulated tyre loads highlighted a roll balance ratio that changes by a factor $\approx 2$ along the circuit; commonly used car models based on quasi steady state loads are not able to capture this effect as long as they assume a constant roll balance ratio. Further work can study more in detail the differences in lap time and car optimisation that arise when using common quasi-steady state car models. Finally, this car model can be extended to a Formula 1 car, simply adding KERS system modelling.

\section{References}

[1] Candelpergher A, Gadola M, Vetturi D. Developments of a method for lap time simulation. SAE Technical Paper; 2000.

[2] Brayshaw D, Harrison M. A quasi steady state approach to race car lap simulation in order to understand the effects of racing line and centre of gravity location. Proceedings of the Institution of Mechanical Engineers, Part D: Journal of Automobile Engineering. $2005 ; 219(6): 725-739$.

[3] Siegler B, Deakin A, Crolla D. Lap time simulation: Comparison of steady state, quasistatic and transient racing car cornering strategies. SAE Technical Paper; 2000.

[4] Blasco-Figueroa J. Minimum Time Manoeuvre Based in the GG-Speed Envelope. Master's thesis, School of Engineering, Cranfield University. 2000;168.

[5] www.ansibledesign.com//dlts//dlts_home.htm; 0000.

[6] Casanova D. On minimum time vehicle manoeuvring: The theoretical optimal lap. Cranfield University; 2000.

[7] Casanova D, Sharp RS, Symonds P. Minimum time manoeuvring: The significance of yaw inertia. Vehicle system dynamics. 2000;34(2):77-115.

[8] Casanova D, Sharp RS, Symonds P. On the optimisation of the longitudinal location of the mass centre of a Formula One car for two circuits. In: Proceedings of AVEC. vol. 2; 2002. p. $6-12$.

[9] Kelly DP. Lap time simulation with transient vehicle and tyre dynamics. Cranfield University; 2008.

[10] Perantoni G, Limebeer DJ. Optimal control for a formula one car with variable parameters. Vehicle System Dynamics. 2014;52(5):653-678. 
[11] Limebeer D. Optimising the Aero-suspension Interactions in a Forumla One car. IEEE Transactions on Control Systems Technology. 2014 8;.

[12] Limebeer DJ, Perantoni G, Rao A. Optimal control of Formula One car energy recovery systems. International Journal of Control. 2014;87(10):2065-2080.

[13] Tremlett A, Limebeer D. Optimal tyre usage for a Formula One car. Vehicle System Dynamics. 2016;54(10):1448-1473.

[14] Kelly DP, Sharp RS. Time-optimal control of the race car: influence of a thermodynamic tyre model. Vehicle System Dynamics. 2012;50(4):641-662.

[15] Tavernini D, Massaro M, Velenis E, Katzourakis DI, Lot R. Minimum time cornering: the effect of road surface and car transmission layout. Vehicle System Dynamics. 2013;51(10):1533-1547.

[16] Tavernini D, Velenis E, Lot R, Massaro M. The Optimality of the Handbrake Cornering Technique. Journal of Dynamic Systems, Measurement, and Control. 2014;136(4):041019.

[17] Pacejka H. Tire and vehicle dynamics. Elsevier; 2006.

[18] Lot R, Dal Bianco N. The significance of high-order dynamics in lap time simulations. In: Proceedings of the 24th Symposium of the International Association for Vehicle System Dynamics, IAVSD, Graz; 2015. .

[19] Bryson AE. Dynamic optimization. vol. 1. Prentice Hall; 1999.

[20] Pinch ER. Optimal control and the calculus of variations. Oxford University Press Oxford; 1993.

[21] Betts JT. Practical methods for optimal control and estimation using nonlinear programming. vol. 19. Siam; 2010.

[22] Bertolazzi E, Biral F, Da Lio M. Symbolic-numeric indirect method for solving optimal control problems for large multibody systems. Multibody System Dynamics. 2005;13(2):233252 .

[23] Biral F, Bertolazzi E, Bosetti P. Notes on numerical methods for solving optimal control problems. IEEJ Journal of Industry Applications. 2016;5(2):154-166.

[24] Benini C, Gadola M, Chindamo D, Uberti S, Marchesin FP, Barbosa RS. The influence of suspension components friction on race car vertical dynamics. Vehicle System Dynamics. 2017;55(3):338-350.

[25] Marchesin BRSAMALGMCDBC F P. Upright mounted pushrod: The effects on racecar handling dynamics. In: Proceedings of the 24th Symposium of the International Association for Vehicle System Dynamics, IAVSD, Graz; 2015. .

[26] Denavit J. A kinematic notation for lower-pair mechanisms based on matrices. Trans of the ASME Journal of Applied Mechanics. 1955;22:215-221.

[27] Lot R, Da Lio M. A symbolic approach for automatic generation of the equations of motion of multibody systems. Multibody System Dynamics. 2004;12(2):147-172.

[28] Pirelli GP2 official tyre data 2011-2015;.

[29] Lot R, Biral F. A Curvilinear Abscissa Approach for the Lap Time Optimization of Racing Vehicles. In: World Congress. vol. 19; 2014. p. 7559-7565. 
[30] Viviani P, Flash T. Minimum-jerk, two-thirds power law, and isochrony: converging approaches to movement planning. Journal of Experimental Psychology: Human Perception and Performance. 1995;21(1):32.

[31] Biral F, Da Lio M, Bertolazzi E. Combining safety margins and user preferences into a driving criterion for optimal control-based computation of reference maneuvers for an ADAS of the next generation. In: Intelligent Vehicles Symposium, 2005. Proceedings. IEEE. IEEE; 2005. p. 36-41.

[32] Bosetti P, Da Lio M, Saroldi A. On the human control of vehicles: an experimental study of acceleration. European Transport Research Review. 2014;6(2):157-170.

[33] Lot R, Dal Bianco N. Lap time optimisation of a racing go-kart. Vehicle System Dynamics. 2015;p. 1-21.

[34] Bertolazzi E, Biral F, Da Lio M. Symbolic-numeric efficient solution of optimal control problems for multibody systems. Journal of computational and applied mathematics. 2006;185(2):404-421. 


\section{Appendix A Suspension kinematics}

In a GP2 car the suspension system is based on short-long arm (SLA) type, as shown in figure 9: the wheel is connected to the chassis by means of two A-shaped arms that end with spherical joints, plus the steering rod which ends either in a movable spherical joint, in the case of the front suspension, or in a fixed spherical joint, in the case of the rear one (even if the rear wheels have no steer, we will refer to this latter rod as "steering rod", as for the front suspension). From the kinematic point of view, the SLA suspension is a special case of the multilink suspension and it is composed by five rods attached through spherical joints to the chassis at one edge and to the wheel plate at the other edge. With reference to figure 9, attachments points fixed to the vehicle frame and are named: upper front chassis point $\mathbf{C}^{1}$, lower front chassis point $\mathbf{C}^{2}$, upper rear chassis point $\mathbf{C}^{3}$, lower rear chassis point $\mathbf{C}^{4}$ and steer chassis point $\mathbf{C}^{5}$. This latter point, which is connected to the steering rod, is fixed to the chassis in the case of the rear suspension, while in the front one it moves along the y-axis as the driver steers. On the wheel side, attachments points are named: upper front wheel point $\mathbf{P}^{1}$, lower front wheel point $\mathbf{P}^{2}$, upper rear wheel point $\mathbf{P}^{3}$, lower rear wheel point $\mathbf{P}^{4}$ and steer wheel point $\mathbf{P}^{5}$. Due to the design of this SLA suspensions, the point $\mathbf{P}^{1}$ coincide with $\mathbf{P}^{3}$, and $\mathbf{P}^{2}$ with $\mathbf{P}^{4}$ (see figure 9).

The wheel plate position and orientation can be completely described by the displacement of the wheel plate centre w.r.t. its nominal configuration $\left(x_{a s}, y_{a s}, z_{a s}\right)$, the steering angle $\delta_{a s}$, the camber angle $\gamma_{a s}$ and the spin angle $\mu_{a s}$ :

$$
\boldsymbol{w}_{a s}=\left\{x_{a s}, y_{a s}, z_{a s}, \gamma_{a s}, \mu_{a s}, \delta_{a s}\right\}
$$

The rigid motion of the wheel is described by the $4 \times 4$ transformation matrix method (27) as follows:

$$
\begin{aligned}
\boldsymbol{W}\left(\boldsymbol{w}_{a s}\right) & =\boldsymbol{W}_{a s 0} \boldsymbol{T}\left(x_{a s}, y_{a s}, z_{a s}\right) \boldsymbol{R}_{z}\left(\delta_{a s}\right) \boldsymbol{R}_{x}\left(\gamma_{a s}\right) \boldsymbol{R}_{y}\left(\mu_{a s}\right) \\
& \equiv \boldsymbol{W}_{a s 0} \boldsymbol{S}\left(\boldsymbol{w}_{a s}\right)
\end{aligned}
$$

where $\boldsymbol{W}_{a s 0}$ is the wheel reference system in nominal conditions, $\boldsymbol{T}\left(x_{a s}, y_{a s}, x_{a s}\right)$ is the translation transformation matrix, and $\boldsymbol{R}_{i}(a)$ are the rotation matrices around $i$-axis of an angle a. From the equivalence in (33), $\boldsymbol{S}\left(\boldsymbol{w}_{a s}\right)$ is the transformation matrix that gives the wheel plate configuration with respect to its nominal position. Since the suspension linkages allow the wheel to have only one degree of freedom, the coordinates $\boldsymbol{w}$ are mutually dependent and can be expressed as function of only one independent parameter; the wheel plate vertical displacement $z_{a s}$ has been chosen as independent dof since it represent the most important movement of the suspension. The five mathematical constraints necessary to remove the dependent variables can be obtained by imposing that the distance between the connecting points located at the extremities of each rod must be equal to the rod length. As the wheel plate moves, the coordinates of each connecting point on the wheel side $\boldsymbol{P}^{i}=\left\{x_{P^{i}}, y_{P^{i}}, z_{P^{i}}, 1\right\}^{T}$ can be easily calculated from their nominal position $\boldsymbol{P}_{0}^{i}$ :

$$
\boldsymbol{P}^{i}=\boldsymbol{W}_{a s 0} \boldsymbol{S}\left(\boldsymbol{w}_{a s}\right) \boldsymbol{W}_{a s 0}^{-1} \boldsymbol{P}_{0}^{i}
$$

Therefore the five constraints can be expressed by the following relationship:

$$
\left(x_{P^{i}}-x_{C^{i}}\right)^{2}+\left(y_{P^{i}}-y_{C^{i}}\right)^{2}+\left(z_{P^{i}}-z_{C^{i}}\right)^{2}-l_{i}^{2}=0, \quad i \in\{1 . .5\},
$$

where $l_{i}$ is the length of the rod connecting $\boldsymbol{P}^{i}$ with $\boldsymbol{C}^{i}$. In conclusion, a set of five algebraic constraint equations is obtained for each suspension:

$$
\phi_{i}\left(\boldsymbol{w}_{a s}\right)=0, \quad i \in 1 . .5
$$

These constraint equations have been solved numerically for the given suspension geometry, both rear and front.

The displacement of the wheel centre $x_{r r}, y_{r r}$, as well as camber $\gamma_{r r}$, and steer $\delta_{r r}$ angles of the rear right wheel plate are depicted as a function of the vertical travel $z_{r r}$ in figure 10a. 
The figure shows that the wheel plate translation along $x$ and $y$ direction are less than $1 \mathrm{~mm}$, moreover the camber and steer angles are smaller than $0.5^{\circ}$ and $0.02^{\circ}$. The trend of $x_{r r}, y_{r r}, \gamma_{r r}$ and $\delta_{r r}$ as function of $z_{r r}$ has been fitted by polynomials up to the second order, and the resulting fittings are shown in figure 10a by continuous lines. The fitting of such variables is required to analytically express the velocity ratio between the wheel plate movements and the vertical displacement $z_{r r}$, as required in equation (7). In the front suspension the position of the steering chassis point $\left(\mathbf{P}^{5}\right)$ along the $y$-axis is controlled by the driver input on the steering wheel, therefore the movements of the front wheel plate $\left(x_{f s}, y_{f s}, \gamma_{f s}, \delta_{f s}\right)$ depend both on the wheel vertical shift $z_{f s}$ and on the steering rack lateral displacement $y_{s}$. Figure 10b shows the front right wheel motion as function of $z_{f r}$ when the steering is null, while figure 10c shows the same quantities as function of $y_{s}$ when the suspension travel is zero. Similarly to the rear suspension, the variables $x_{f r}, y_{f r}, \gamma_{f r}, \delta_{f r}$ have been fitted by polynomials up to the second order as function of $z_{f r}$ and $y_{s}$, and the resulting fits are shown in the figures by continuous lines. The same procedure described just above also allows calculation of the reduced stiffness at wheel of the torsion bars, coil springs, dampers and anti-roll bars. The rear wheels are connected through a push-rod and a rocker to the dampers and the coil springs, as shown in figure 9. The rear anti-roll bar is connected to the left and right rocker through two link-bars. In the front suspensions, again, a push-rod connects the wheel to a rocker; however, this rocker is not free to rotate around its pivot because it is connected to the chassis with a torsion bar. Thus, when the suspension moves, the elastic force is exerted by this torsion bar. Then, the damper and the anti-roll bar are attached to the rocker similarly to the rear suspension design. The travel of the dampers, rear coil springs and front torsion bars has again been fitted by polynomials as functions of $z_{a s}$ and $y_{r}$, and the resulting polynomial coefficients are reported in table 1

\begin{tabular}{cl}
\hline variable and fit & description \\
\hline$\xi_{r s}=-0.832 z_{r s}$ & rear dampers and coil springs travel \\
$\xi_{f r}=-0.879 z_{f r}+0.0662 y_{r}$ & front right damper travel \\
$\xi_{f l}=-0.879 z_{f l}-0.0662 y_{r}$ & front left damper travel \\
$\theta_{f r}=-0.605 z_{f r}+0.0458 y_{r}$ & front right torsion bar angle ${ }^{1}$ \\
$\theta_{f l}=-0.605 z_{f l}-0.0458 y_{r}$ & front left torsion bar angle ${ }^{1}$ \\
\hline
\end{tabular}

Table 1: Spring and damper travels. Ranges are: $-20 \mathrm{~mm}<z_{a s}<20 \mathrm{~mm},-14 \mathrm{~mm}<y_{r}<$ $14 \mathrm{~mm} .{ }^{1}$ the coefficients are expressed in degrees per millimetre. 


\section{Appendix B List of symbols}

\begin{tabular}{|c|c|c|}
\hline degrees of freedom & units & description \\
\hline$V$ & $\mathrm{~m} / \mathrm{s}$ & speed \\
\hline$\lambda$ & $\mathrm{rad}$ & drift angle \\
\hline$z$ & $\mathrm{~m}$ & centre of mass (CoM) vertical displacement \\
\hline$\phi$ & $\mathrm{rad}$ & roll angle \\
\hline$\mu$ & $\mathrm{rad}$ & pitch angle \\
\hline$\Omega_{z}$ & $\mathrm{rad} / \mathrm{s}$ & yaw rate \\
\hline$z_{a s}$ & $\mathrm{~m}$ & wheel vertical displacement with respect to the chassis \\
\hline$\omega_{a s}$ & $\mathrm{rad} / \mathrm{s}$ & wheel spin velocity \\
\hline dependent variables & units & description \\
\hline$y_{r}$ & $\mathrm{~m}$ & steering rack displacement \\
\hline$\xi_{\text {as }}$ & $\mathrm{m}$ & tyre radial deformation \\
\hline$h_{f}$ & $\mathrm{~m}$ & front ride height \\
\hline$h_{r}$ & $\mathrm{~m}$ & rear ride height \\
\hline$s$ & $\mathrm{~m}$ & road centre line curvilinear abscissa \\
\hline$n$ & $\mathrm{~m}$ & car lateral displacement from road centre line \\
\hline$\alpha$ & $\mathrm{rad}$ & car heading relative to road centre line \\
\hline$\kappa$ & $m^{-1}$ & road curvature \\
\hline$\psi_{a s}$ & rad & wheel steering angle \\
\hline$\phi_{a s}$ & $\mathrm{rad}$ & wheel camber angle \\
\hline$x_{a s}$ & $\mathrm{~m}$ & $\begin{array}{l}\text { wheel plate displacement along x-axis } \\
\text { (with respect to nominal position) }\end{array}$ \\
\hline$y_{a s}$ & $\mathrm{~m}$ & $\begin{array}{l}\text { wheel plate displacement along y-axis } \\
\text { (with respect to nominal position) }\end{array}$ \\
\hline$\gamma_{a s}$ & $\mathrm{rad}$ & wheel plate camber angle (with respect to nominal position) \\
\hline$\mu_{a s}$ & rad & wheel plate pitch angle (with respect to nominal position) \\
\hline$\delta_{a s}$ & $\mathrm{rad}$ & wheel plate steering angle (with respect to nominal position) \\
\hline$N_{a s}$ & $\mathrm{~N}$ & tyre load \\
\hline$S_{a s}$ & $\mathrm{~N}$ & tyre longitudinal force \\
\hline$F_{a s}$ & $\mathrm{~N}$ & tyre lateral force \\
\hline$T_{e}$ & $\mathrm{Nm}$ & engine torque \\
\hline$T_{b}$ & $\mathrm{Nm}$ & braking torque \\
\hline$T_{a s}$ & $\mathrm{Nm}$ & driving torque applied to the wheel \\
\hline$J_{a s}$ & $\mathrm{~N}$ & suspension force acting on the chassis and counter-reacting on \\
\hline$D$ & $\mathrm{~N}$ & drag force \\
\hline$L_{f}$ & $\mathrm{~N}$ & front axle aero-downforce \\
\hline$L_{r}$ & $\mathrm{~N}$ & rear axle aero-downforce \\
\hline$R$ & $\mathrm{~N}$ & tyres rolling resistance \\
\hline$\kappa_{a s}$ & & tyre longitudinal slip \\
\hline$\lambda_{a s}$ & $\mathrm{rad}$ & tyre sideslip angle \\
\hline$V_{a s}^{c p}$ & $\mathrm{~m} / \mathrm{s}$ & tyre contact point longitudinal speed \\
\hline$V_{a s}^{r}$ & $\mathrm{~m} / \mathrm{s}$ & tyre contact point spinning speed \\
\hline$V_{a s}^{l}$ & $\mathrm{~m} / \mathrm{s}$ & tyre contact point lateral speed \\
\hline
\end{tabular}


Table 2: Model variables. The suffix $a \in\{r, f\}$ stands for rear (r) or front (f) axle, while the suffix $s \in\{r, l\}$ stand for right (r) or left (l) side.

\section{Appendix C Vehicle data}

\begin{tabular}{|c|c|c|c|}
\hline symbol & value & units & description \\
\hline$g$ & 9.81 & $m / s^{2}$ & gravitational acceleration \\
\hline$\rho$ & 1.2 & $\mathrm{~kg} / \mathrm{m}^{3}$ & air density \\
\hline$h$ & 0.31 & $m$ & centre of gravity (CoG) height \\
\hline$b_{r}=b_{r r}=b_{r l}$ & 1.34 & $m$ & $\mathrm{x}$-axis distance between the rear axle and the vehicle \\
\hline$b_{f}=-b_{f r}=-b_{f l}$ & 1.78 & $m$ & $\begin{array}{l}\text { CoG } \\
\text { x-axis distance between the front axle and the vehicle } \\
\text { CoG }\end{array}$ \\
\hline$w$ & 3.12 & $m$ & wheelbase \\
\hline$t_{f}=t_{f l}=-t_{f r}$ & 0.739 & $m$ & front half track \\
\hline$t_{r}=t_{r l}=-t_{r r}$ & 0.708 & $m$ & rear half track \\
\hline$h_{r 0}$ & $6 \mathrm{e}-2$ & $m$ & nominal rear ride height \\
\hline$h_{f 0}$ & $2 \mathrm{e}-2$ & $m$ & nominal front ride height \\
\hline$\beta$ & 0.62 & & front braking bias \\
\hline$\delta_{r r 0}$ & -0.04 & $\operatorname{deg}$ & rear right wheel nominal steer angle \\
\hline$\gamma_{r r 0}$ & -0.5 & deg & rear right wheel nominal camber angle \\
\hline$\delta_{f r 0}$ & 0.09 & deg & front right wheel nominal steer angle \\
\hline$\gamma_{f r 0}$ & -2.7 & $\operatorname{deg}$ & front right wheel nominal camber angle \\
\hline$m$ & 700 & $\mathrm{~kg}$ & vehicle mass (rider included) \\
\hline$I_{x x}$ & 200 & $\mathrm{kgm}^{2}$ & roll moment of inertia \\
\hline$I_{y y}$ & 1000 & $\mathrm{kgm}^{2}$ & pitch moment of inertia \\
\hline$I_{z z}$ & 1100 & $\mathrm{kgm}^{2}$ & yaw moment of inertia \\
\hline$I_{x z}$ & 0 & $\mathrm{kgm}^{2}$ & mixed moment of inertia \\
\hline$m_{r r}=m_{r l}$ & 30.4 & $k g$ & rear wheel mass \\
\hline$m_{f r}=m_{f l}$ & 24.4 & $\mathrm{~kg}$ & front wheel mass \\
\hline$I_{r r}=I_{r l}$ & 1.55 & $\mathrm{kgm}^{2}$ & rear wheel spin inertia (including half of rear axle) \\
\hline$I_{r r}=I_{r l}$ & 1 & $\mathrm{kgm}^{2}$ & front wheel spin inertia \\
\hline$n_{d r}$ & $2.03 \mathrm{e} 5$ & $N / m$ & rear tyre load coefficient \\
\hline$n_{d f}$ & $1.96 \mathrm{e} 5$ & $N / m$ & front tyre load coefficient \\
\hline$n_{\text {dor }}$ & $7.28 \mathrm{e} 2$ & $N s / m$ & rear tyre load coefficient \\
\hline$n_{\text {dof }}$ & $4.73 \mathrm{e} 2$ & $N s / m$ & front tyre load coefficient \\
\hline$n_{o r}$ & $5.85 \mathrm{e}-2$ & $N s^{2}$ & rear tyre load coefficient \\
\hline$n_{o f}$ & $1.57 \mathrm{e}-2$ & $N s^{2}$ & front tyre load coefficient \\
\hline$n_{y r}$ & $-1.39 \mathrm{e}-5$ & $N^{-1}$ & rear tyre load coefficient \\
\hline$n_{y f}$ & $-1.9 \mathrm{e}-5$ & $N^{-1}$ & front tyre load coefficient \\
\hline$n_{c r}$ & 500 & $N s / m$ & rear tyre load damping stiffness \\
\hline$n_{c f}$ & 500 & $N s / m$ & front tyre load damping stiffness \\
\hline$\rho_{r}$ & 0.01 & & rear tyre rolling resistance coefficient \\
\hline$\rho_{f}$ & 0.01 & & front tyre rolling resistance coefficient \\
\hline$r_{r}$ & 0.31 & $m$ & rear tyres radius \\
\hline
\end{tabular}




\begin{tabular}{llll}
$r_{f}$ & 0.31 & $m$ & front tyres radius \\
& & & \\
$\sigma_{y}$ & 0.1 & $m$ & tyres lateral relaxation length \\
$\sigma_{x}$ & 0.1 & $m$ & tyres longitudinal relaxation length \\
\hline
\end{tabular}

Table 3: Vehicle parameters.

\section{List of Figures}

1 Car chassis degrees of freedom $($ dof $) \ldots \ldots \ldots \ldots \ldots$

2 Road tracking: the curvilinear abscissa $s$, car lateral displacement from road centre line $n$ and car relative heading angle $\alpha$ are displayed. . . . . . . . . . . 9

3 Optimal trajectory on Montmelò circuit. Numbers within ochre boxes indicate the value of the road centre line abscissa in meters. . . . . . . . . . . . . . . . 12

4 Speed (top), longitudinal (centre) and lateral (bottom) accelerations versus distance travelled. Blue continuous lines refer to simulation outcomes, orange dashed ones to telemetry data. . . . . . . . . . . . . . . . . . 22

5 Accelerations versus car speed (top left and bottom right) and g-g diagram (bottom left). Blue crosses refer to simulation outcomes, orange circles to telemetry data. The car model reproduces well the real accelerations envelope, moreover the increase of accelerations bounds with speed due to aerodynamic down-force is highly noticeable and well captured. . . . . . . . . . . . . . . . 23

6 From top to bottom: simulated speed (a), chassis roll $\phi$ and pitch $\mu$ angles (b), chassis vertical displacement $z$ (c), aerodynamic down-forces $L_{f}+L_{r}$ and drag

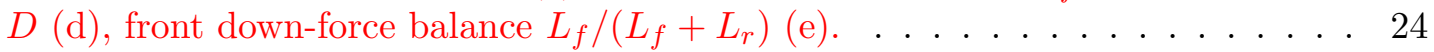

7 Simulated suspension travels, tyre radial deformations and tyre loads (left side

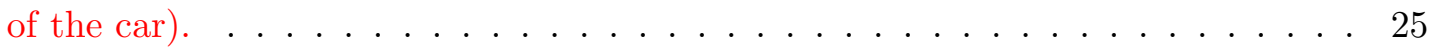

8 Rear axle lateral load transfer $N_{r}^{\text {lat }} \equiv N_{r r}-N_{r l}$ versus front axle one $N_{f}^{l a t} \equiv N_{f r}-$ $N_{f l}$ (blue crosses). The two dashed lines correspond to the linear relationship $N_{r}^{\text {lat }}=1.12 N_{f}^{\text {lat }}$ and $N_{r}^{\text {lat }}=0.66 N_{f}^{\text {lat }}$. It is evident that the roll balance ratio $N_{r}^{\text {lat }} / N_{f}^{\text {lat }}$ varies of a factor of almost 2 along the track. . . . . . . . . . 26

9 Front (left) and rear (right) short-long arm suspension scheme. . . . . . . . . 26

10 Suspension kinematics analysis: rear and front wheel plate motions are shown as function of the suspension degrees of freedom. . . . . . . . . . . . . . . 27 

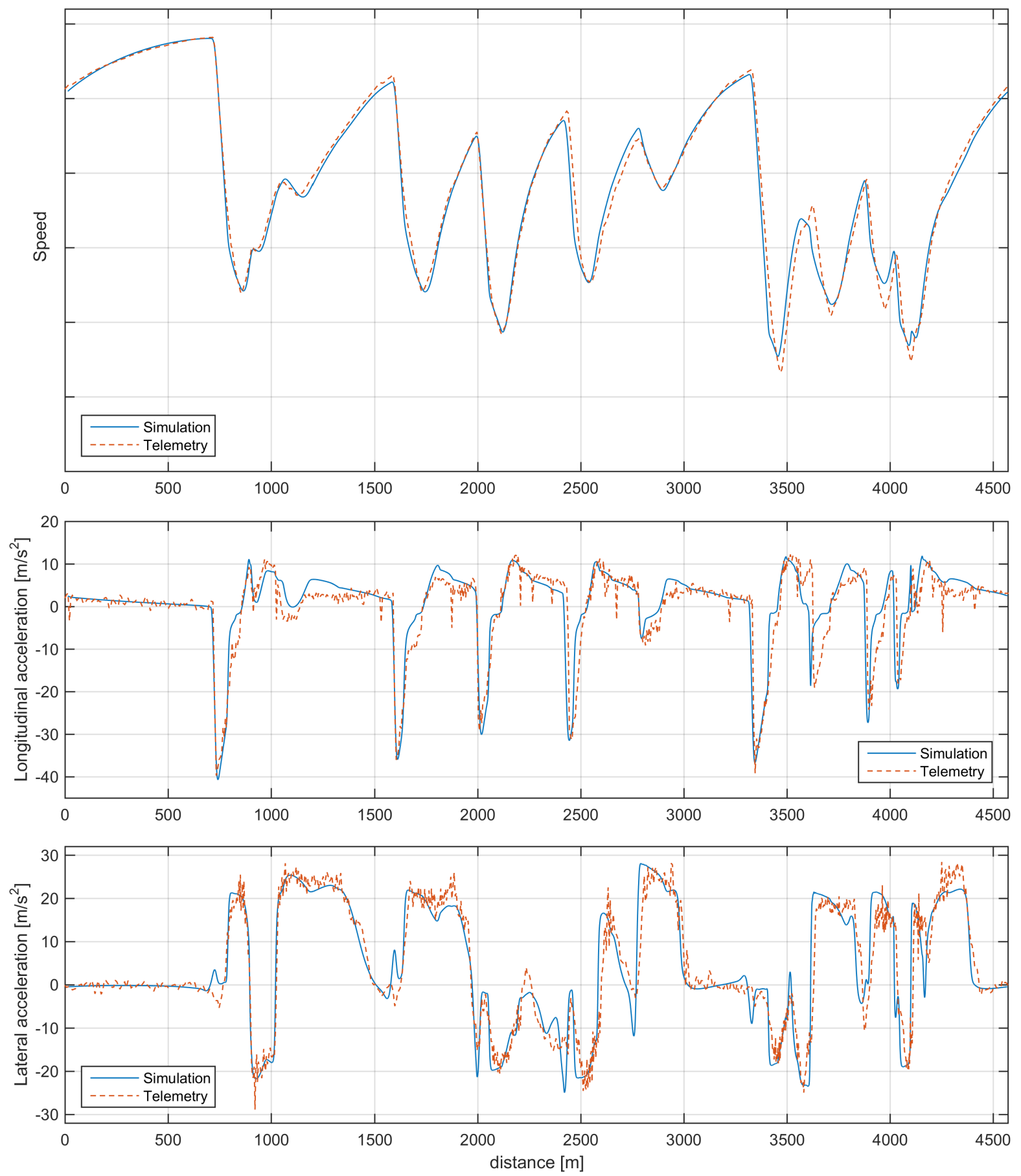

Figure 4: Speed (top), longitudinal (centre) and lateral (bottom) accelerations versus distance travelled. Blue continuous lines refer to simulation outcomes, orange dashed ones to telemetry data. 

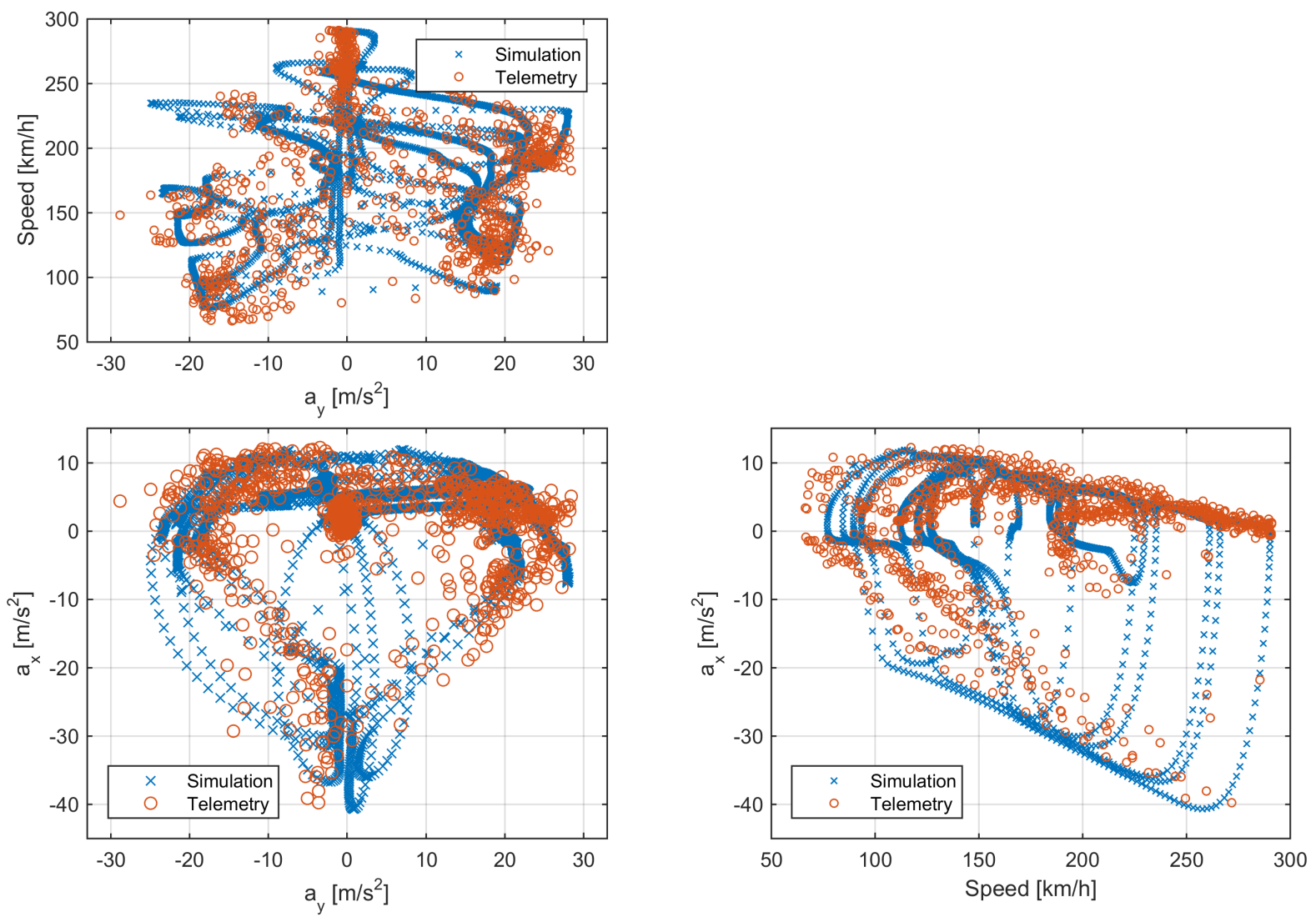

Figure 5: Accelerations versus car speed (top left and bottom right) and g-g diagram (bottom left). Blue crosses refer to simulation outcomes, orange circles to telemetry data. The car model reproduces well the real accelerations envelope, moreover the increase of accelerations bounds with speed due to aerodynamic down-force is highly noticeable and well captured. 
a) Speed

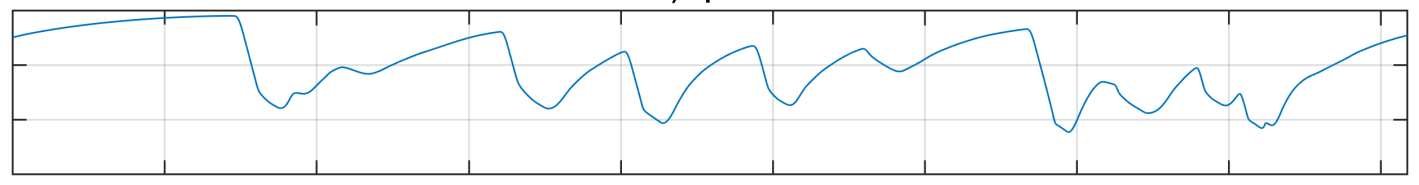

b) Chassis roll and pitch

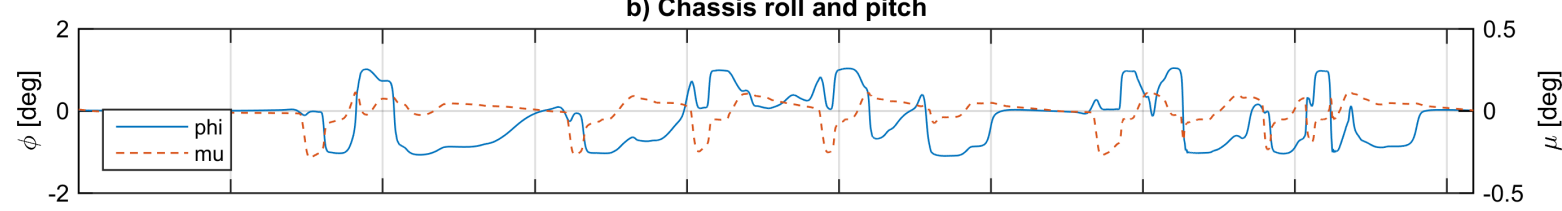

c) Chassis vertical displacement
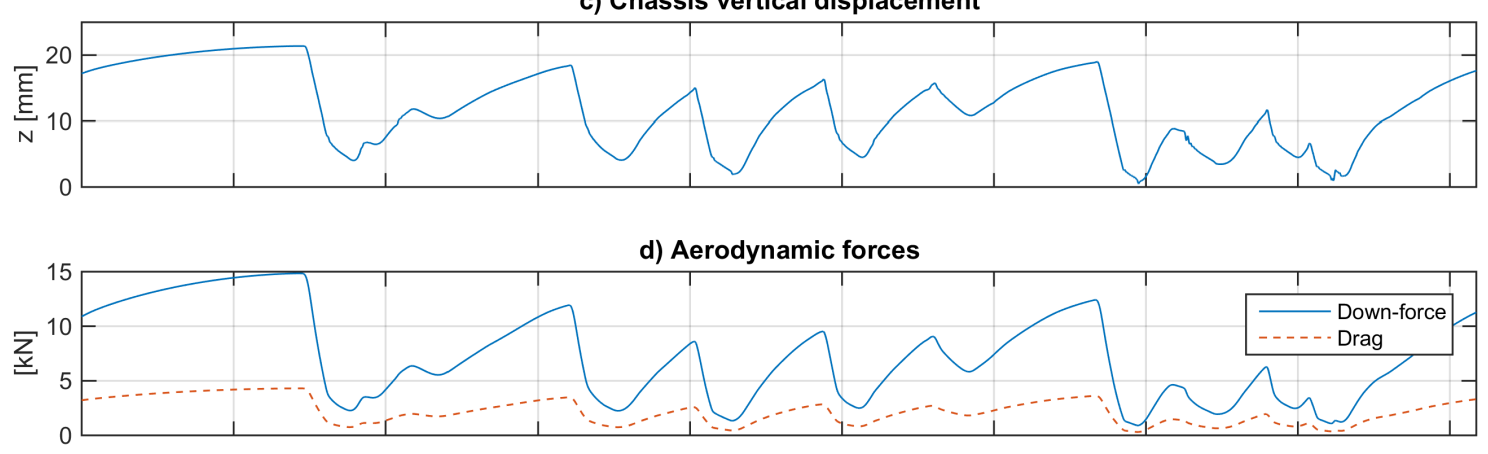

e) Downforce balance

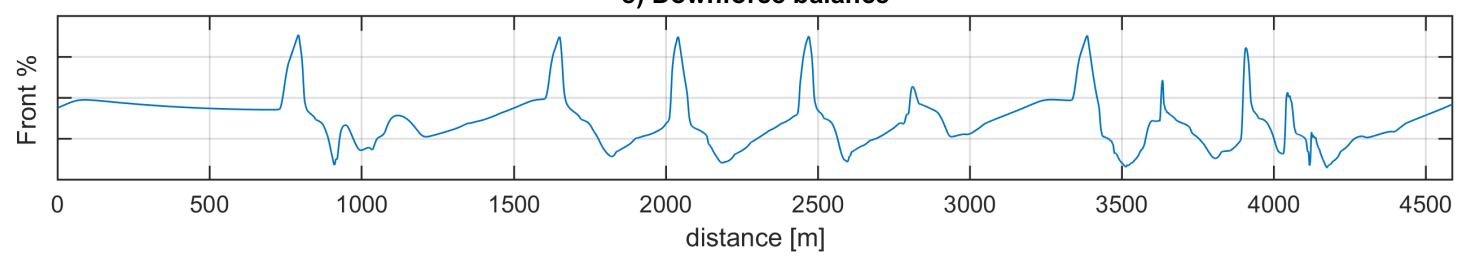

Figure 6: From top to bottom: simulated speed (a), chassis roll $\phi$ and pitch $\mu$ angles (b), chassis vertical displacement $z$ (c), aerodynamic down-forces $L_{f}+L_{r}$ and drag $D(\mathrm{~d})$, front down-force balance $L_{f} /\left(L_{f}+L_{r}\right)(\mathrm{e})$. 
Suspension travels (left side)

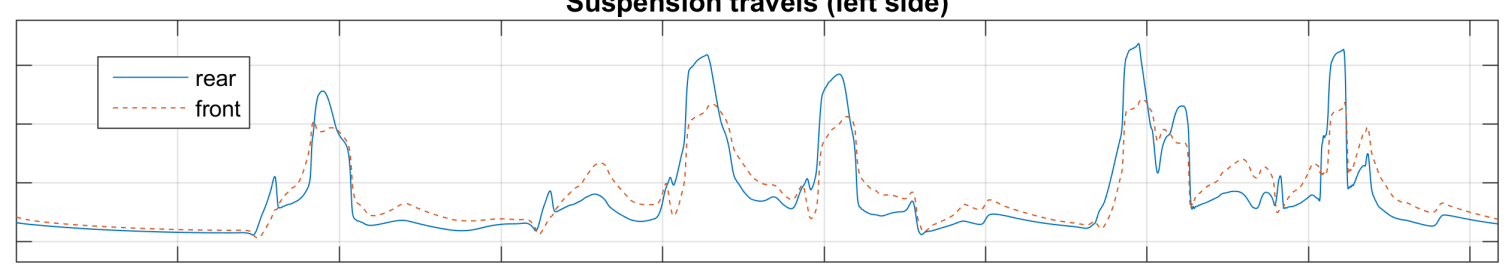

Tyres deformation (left side)

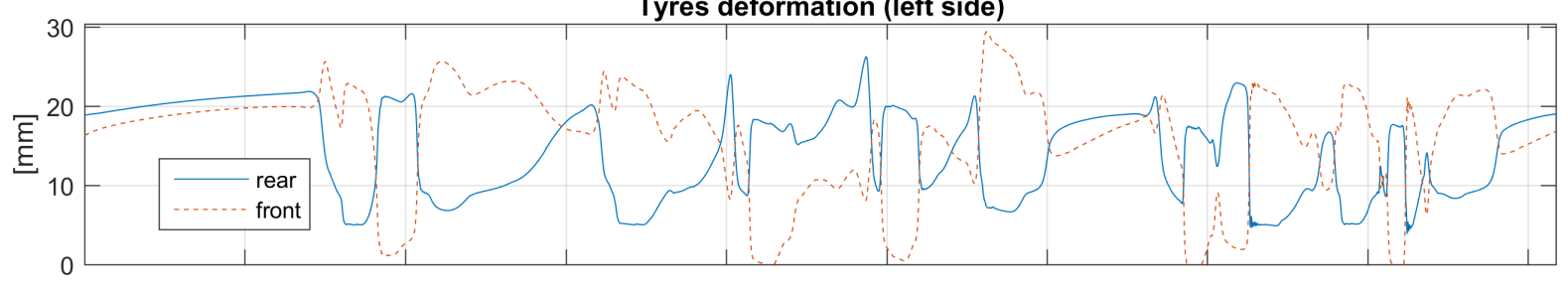

Tyres load (left side)

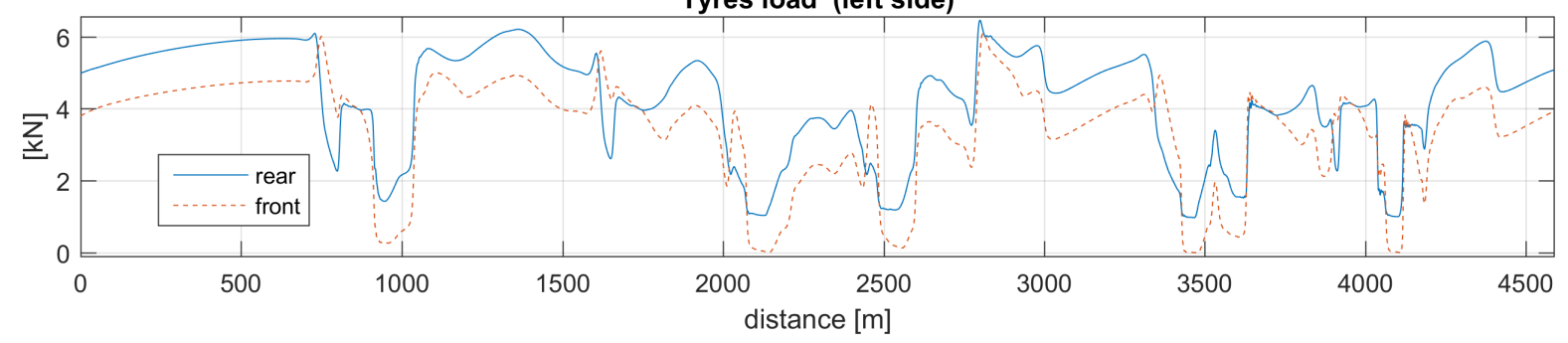

Figure 7: Simulated suspension travels, tyre radial deformations and tyre loads (left side of the car). 


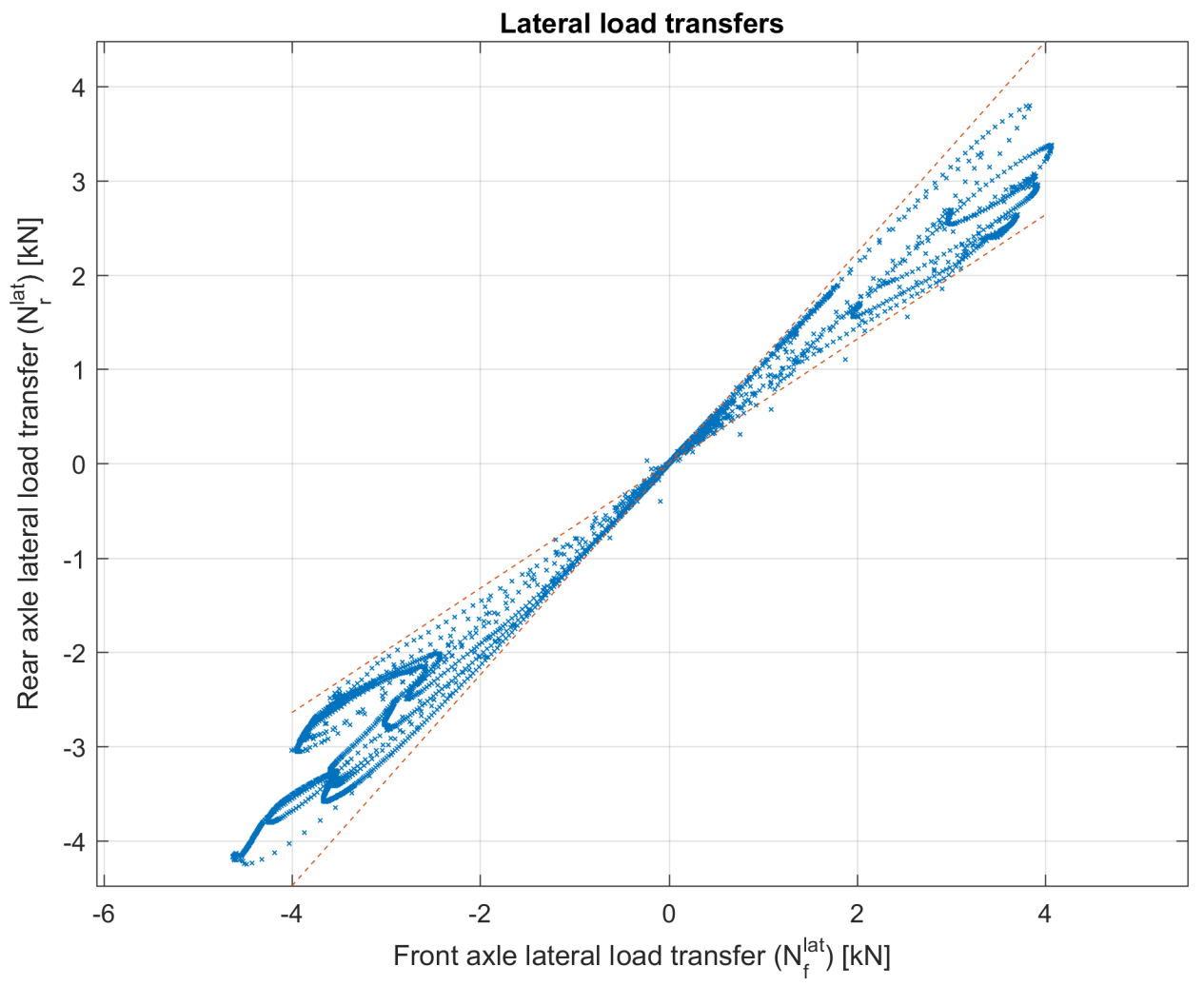

Figure 8: Rear axle lateral load transfer $N_{r}^{l a t} \equiv N_{r r}-N_{r l}$ versus front axle one $N_{f}^{l a t} \equiv N_{f r}-N_{f l}$ (blue crosses). The two dashed lines correspond to the linear relationship $N_{r}^{\text {lat }}=1.12 N_{f}^{\text {lat }}$ and $N_{r}^{l a t}=0.66 N_{f}^{l a t}$. It is evident that the roll balance ratio $N_{r}^{l a t} / N_{f}^{l a t}$ varies of a factor of almost 2 along the track.
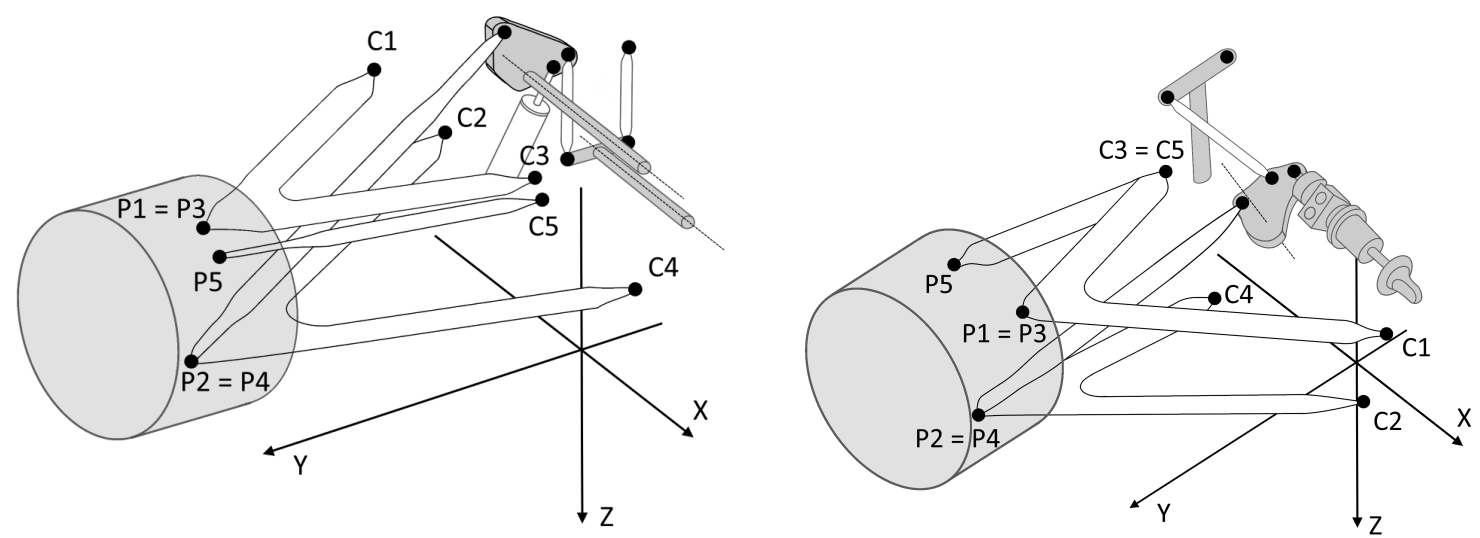

Figure 9: Front (left) and rear (right) short-long arm suspension scheme. 

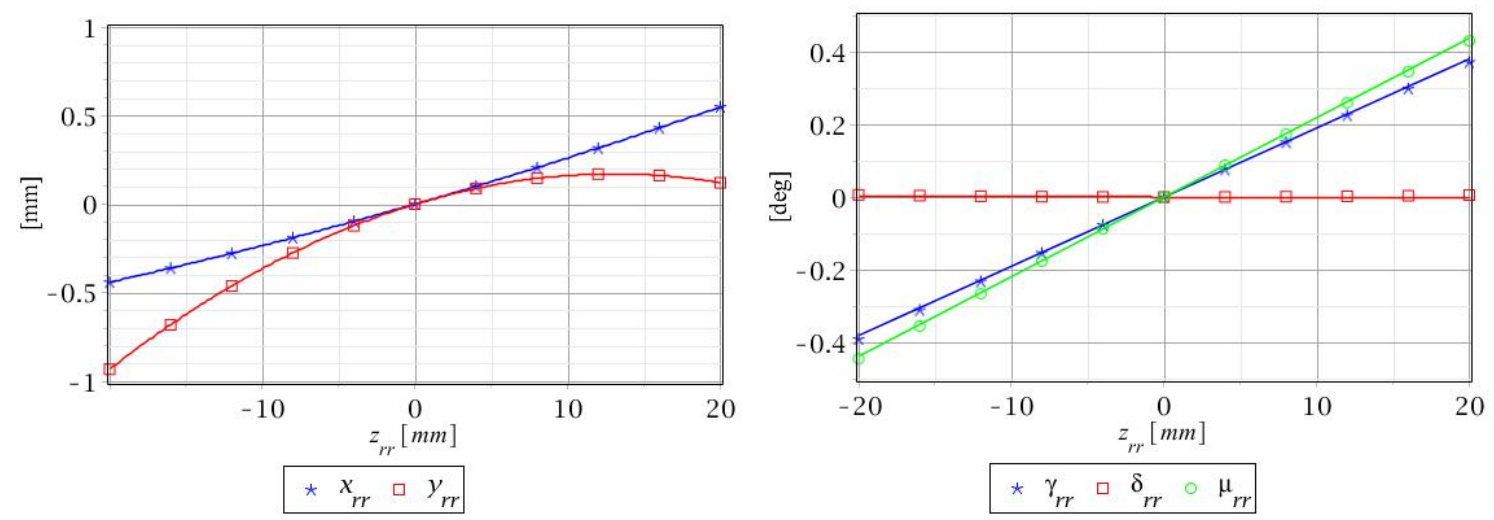

(a) Rear wheel plate movements: $x_{r r}, y_{r r}$ displacements and $\gamma_{r r}, \delta_{r r}$ angles are represented as function of the wheel vertical travel $z_{r r}$. The continuous lines represent the fitting.
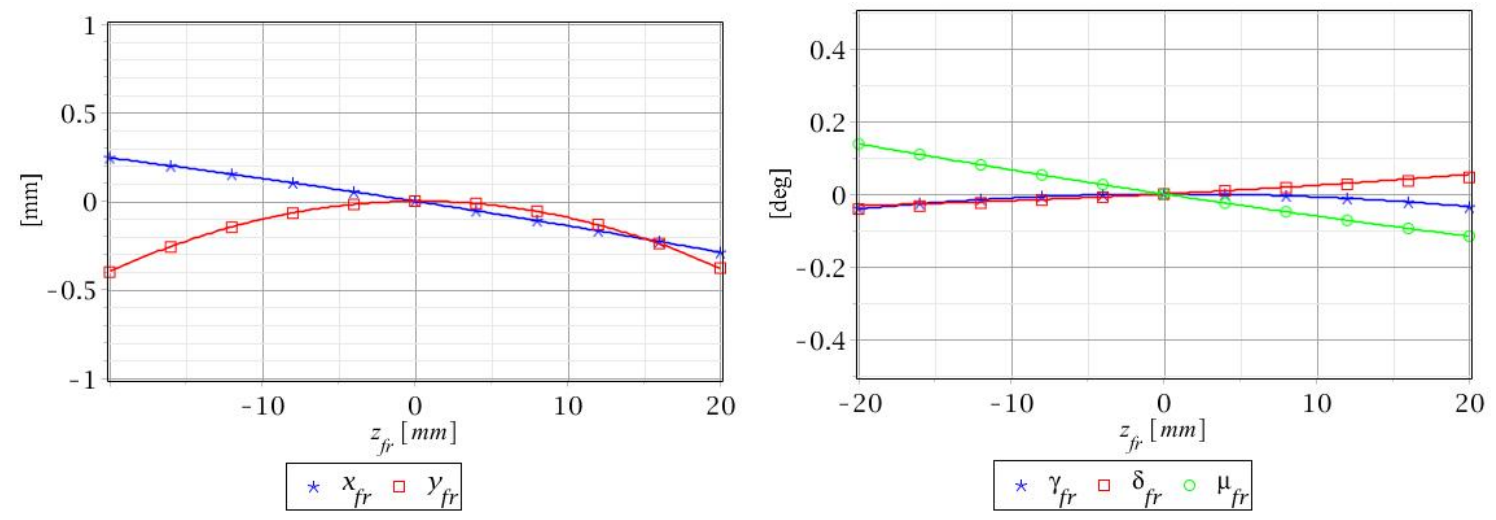

(b) Front wheel plate movements: $x_{f r}, y_{f r}$ displacements and $\gamma_{f r}, \delta_{f r}$ angles are represented as function of the wheel vertical travel $z_{f r}$ when $y_{r}=0$. The continuous lines represent the fitting.
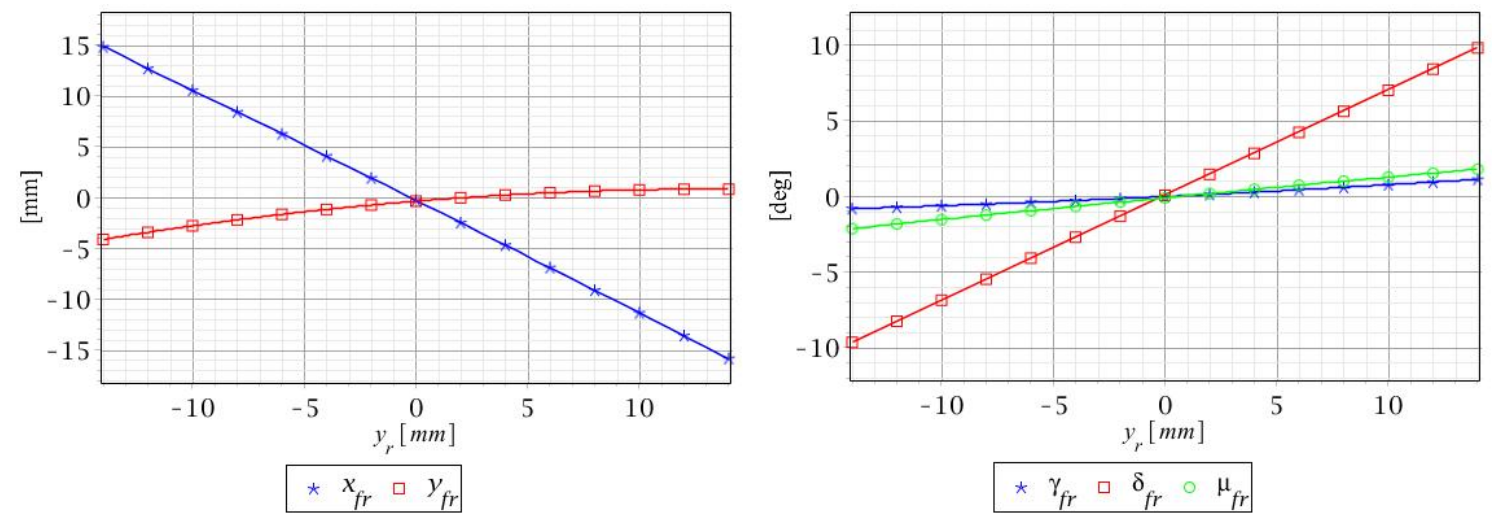

(c) Front wheel plate movements: $x_{f r}, y_{f r}$ displacements and $\gamma_{f r}, \delta_{f r}$ angles are represented as function of the steering rack displacement $y_{r}$ when the vertical displacement is null $z_{f r}=0$. The continuous lines represent the fitting.

Figure 10: Suspension kinematics analysis: rear and front wheel plate motions are shown as function of the suspension degrees of freedom. 